\title{
Synthesis, Chiroptical Behavior, and Sensing of Carboxylic Acid Functionalized Poly(phenylene ethynylene-alt-bithiophene)s
}

\author{
Steven Vandeleene, Michiel Verswyvel, Thierry Verbiest, and Guy Koeckelberghs* \\ Laboratory of Molecular Electronics and Photonics, Katholieke Universiteit Leuven, Celestijnenlaan 200F, \\ 3001 Heverlee (Leuven), Belgium
}

Received August 9, 2010

\begin{abstract}
Several poly(phenylene ethynylene-alt-bithiophene)s with (chiral) nonfunctionalized substituents were synthesized with a variable phenylene ethynylene (PE) spacer length (up to 4 repeating units). The chiroptical behavior was evaluated with UV-vis and circular dichroism (CD) spectroscopy, revealing a highly solvent-sensitive aggregate formation. Based on this high sensitivity, both chiral and achiral carboxylic acid functionalized analogues were prepared, of which the length of the spacer connecting the carboxylic acid to the polymer backbone was varied. A combination of UV-vis, CD, and emission spectroscopy showed a clear affinity of chiral amines toward the functionalized polymers both in solution and in film. However, a different supramolecular behavior of the polymers was observed depending on the length of the carboxylic acid functionalized side chain.
\end{abstract}

\section{Introduction}

The optical, electrical, and electronic properties of conjugated polymers not only depend on their molecular structure but also are to a large extent governed by their conformation and supramolecular structure. A nice example of this phenomenon is the solvatochromism of poly(phenylene ethynylene)s. ${ }^{1}$ Addition of nonsolvent to a solution of this polymer results in a redshift and narrowing of the absorption and fluorescence band, which originates from a planarization and stacking of the polymer chains. In general, the conformation and supramolecular structure can be manipulated by the solvent quality, ${ }^{2}$ the use of additives, ${ }^{3}$ temperature, ${ }^{4}$ etc.

In the case of chiral polymers, the aggregates can be composed of chirally, instead of parallel, stacked coplanar polymer chains, which give rise to chiroptical effects such as circular dichroism. ${ }^{1 b, 5}$ Chirality can easily be implemented by chiral substituents, but also by the use of chiral solvents, media, additives, etc. The binding of a chiral additive can result in an enantioselective and possibly even in a diastereoselective response, as the chiral additive invokes (chiral) aggregation and hence alters the chiroptical properties of the material.

This manuscript describes the synthesis and chiroptical properties of alternating copolymers of oligo(phenylene ethynylene)s (OPE) and 3,3'-dialkoxybithiophene (BT) units. The choice of these building blocks is motivated by the fact that their corresponding homopolymers (poly(phenylene ethynylene) $s^{1}$ and HH-TT coupled poly(3-alkoxythiophene)s ${ }^{6}$ ) are known to adopt a lamellar supramolecular structure in which chirality is strongly expressed. Moreover, the BT units, being electron-rich, can easily be oxidized, allowing the introduction of radical cations confined within the OPE parts. The alternating, regular nature of the copolymer (in contrast to a random copolymer) should allow the formation of a targeted supramolecular behavior. Finally, by the use of two different monomers, one monomer can be equipped with chiral side-chains, while the second monomer can bear a functional group.

*Corresponding author. E-mail: guy.koeckelberghs@chem.kuleuven.be.
The first part of the manuscript deals with the influence of the length of the OPE part on the chiroptical properties of the polymers (P1-4, Figure 1). It is investigated whether the targeted supramolecular behavior is indeed present in these alternating copolymers. In the second part, a carboxylic acid functional group is introduced in the OPE part $(\mathbf{P 5} \mathbf{b}-\mathbf{8 b}$, Figure 1) and the influence of an additive on the chiroptical properties is investigated in detail.

\section{Experimental Section}

Reagents and Instrumentation. All reagents were purchased from Aldrich Chemical Co., Acros Organics, Merck, and Fluka. Reagent grade solvents were dried and purified by distillation.

Gel permeation chromatography (GPC) measurements were done with a Shimadzu 10A apparatus with a tunable absorbance detector and a differential refractometer in tetrahydrofuran (THF) as eluent toward polystyrene standards. ${ }^{1} \mathrm{H}$ nuclear magnetic resonance (NMR) measurements were carried out with a Bruker Avance $300 \mathrm{MHz}$. The IR spectra were obtained with a Bruker ALPHA spectrometer (ATR). UV-vis and CD spectra were recorded with a Varian Cary 400 and a JASCO 62 DS apparatus, respectively. The DSC measurements were performed on a Perkin-Elmer DSC 7 apparatus. The fluorescence measurements were done on a PTI Photon Technology International apparatus. The samples were excited near the absorption wavelength. Films for UV-vis and CD experiments were prepared by spin coating from THF solutions (1200 rpm, $20 \mathrm{~s}$ ).

Compounds $1,{ }^{7} \mathbf{9},{ }^{3 \mathrm{~g}} \mathbf{2 0},{ }^{8} \mathbf{2 1},{ }^{9} \mathbf{2 2},{ }^{6}$ and $\mathbf{2 3}{ }^{6}$ were synthesized according to literature procedures.

Monomer Synthesis. Synthesis of 2.1 (12.6 mmol, $5.91 \mathrm{~g})$, $\mathrm{Pd}\left(\mathrm{PPh}_{3}\right)_{2} \mathrm{Cl}_{2}(0.630 \mathrm{mmol}, 0.442 \mathrm{~g})$, and $\mathrm{PPh}_{3}(0.630 \mathrm{mmol}$, $0.164 \mathrm{~g})$ were added to a mixture of dry THF $(40 \mathrm{~mL})$ and dry diisopropylamine $(50 \mathrm{~mL})$ under argon atmosphere. The solution was heated to $40{ }^{\circ} \mathrm{C}$, followed by the dropwise addition of propargyl alcohol $(14.2 \mathrm{mmol}, 0.791 \mathrm{~g})$ and a solution of $\mathrm{CuI}$ $(1.89 \mathrm{mmol}, 0.356 \mathrm{~g})$ in a mixture of dry THF $(1.5 \mathrm{~mL})$ and dry diisopropylamine $(6.5 \mathrm{~mL})$. The reaction was followed by TLC, and after completion, petroleum ether was added and the mixture was washed with water and filtered. The organic layer 


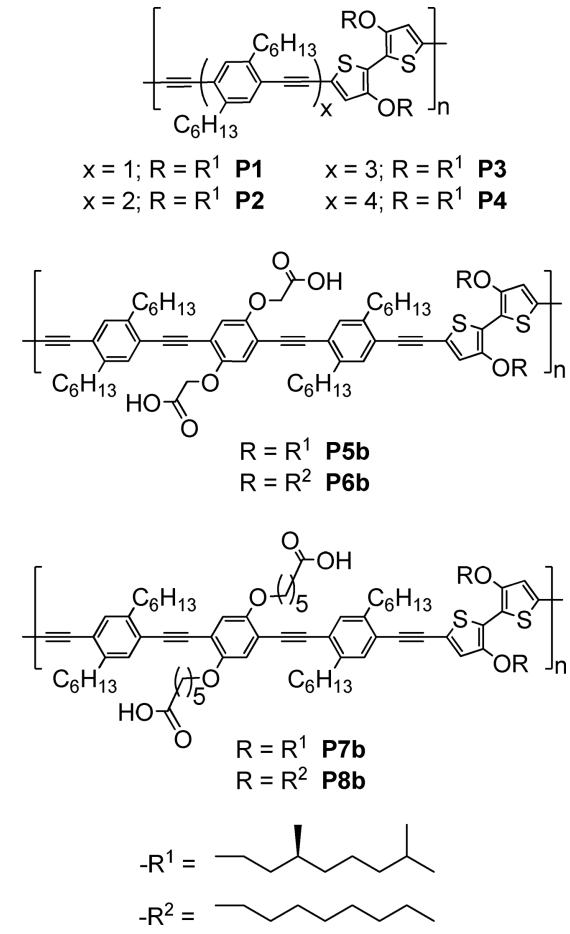

Figure 1. Structure of the polymers.

was washed with a $\mathrm{HCl}$ solution $(1.00 \mathrm{M})$, a saturated $\mathrm{NaHCO}_{3}$ solution, and brine, and finally dried over $\mathrm{MgSO}_{4}$. The solvents were removed under reduced pressure, and the crude product was purified by column chromatography (eluent: dichloromethane). The product was isolated as a brown oil and was used in the next step without further purification. Yield: $4.96 \mathrm{~g}(94 \%) .{ }^{1} \mathrm{H}$ NMR $\left(\mathrm{CD}_{2} \mathrm{Cl}_{2}\right): \delta=7.24(\mathrm{~s}, 1 \mathrm{H}), 7.23(\mathrm{~s}, 1 \mathrm{H}), 4.50(\mathrm{~d}, 2 \mathrm{H}), 2.71-2.65$ $(\mathrm{m}, 4 \mathrm{H}), 1.72(\mathrm{t}, 1 \mathrm{H}), 1.59-1.52(\mathrm{~m}, 4 \mathrm{H}), 1.41-1.21(\mathrm{~m}, 12 \mathrm{H})$, $0.87(\mathrm{~m}, 6 \mathrm{H}), 0.25(\mathrm{~s}, 9 \mathrm{H})$.

Synthesis of $3.2(10.8 \mathrm{mmol}, 4.30 \mathrm{~g})$ was dissolved in a mixture of methanol $(60 \mathrm{~mL})$ and THF $(45 \mathrm{~mL})$ under argon atmosphere. A $\mathrm{NaOH}$ solution $(5.00 \mathrm{M}, 12.0 \mathrm{~mL})$ was added, and the reaction was monitored in TLC. After completion, diethyl ether $(120 \mathrm{~mL})$ and water were added, and the compound was extracted with diethyl ether. The collected organic layers were washed with brine and dried over $\mathrm{MgSO}_{4}$. After removal of the solvent under reduced pressure, the product was purified by column chromatography (eluent: dichloromethane). The product was isolated as a brown oil. Yield: $3.84 \mathrm{~g}(100 \%) .{ }^{1} \mathrm{H}$ NMR $\left(\mathrm{CDCl}_{3}\right): \delta=7.28(\mathrm{~s}, 1 \mathrm{H}), 7.24(\mathrm{~s}, 1 \mathrm{H})$, $4.49(\mathrm{~d}, 2 \mathrm{H}), 3.33(\mathrm{~s}, 1 \mathrm{H}), 2.72-2.65(\mathrm{~m}, 4 \mathrm{H}), 1.65-1.52(\mathrm{~m}, 4 \mathrm{H})$, $1.38-1.20(\mathrm{~m}, 12 \mathrm{H}), 0.87(\mathrm{~m}, 6 \mathrm{H})$.

Synthesis of 4 . The same procedure as described for the synthesis of $\mathbf{2}$ was used, starting from 1 ( $0.876 \mathrm{mmol}, 861 \mathrm{mg})$. The pure product was purified by column chromatography (eluent: dichloromethane) and isolated as a brown oil. Yield: $3.30 \mathrm{~g}(60 \%)$. ${ }^{1} \mathrm{H}$ NMR $\left(\mathrm{CD}_{2} \mathrm{Cl}_{2}\right): \delta=7.32(\mathrm{~s}, 1 \mathrm{H}), 7.31(\mathrm{~s}, 1 \mathrm{H}), 7.28(\mathrm{~s}, 2 \mathrm{H})$, $4.50(\mathrm{~d}, 2 \mathrm{H}), 2.80-2.69(\mathrm{~m}, 8 \mathrm{H}), 1.73(\mathrm{t}, 1 \mathrm{H}), 1.67-1.57(\mathrm{~m}, 8 \mathrm{H})$, $1.48-1.28(\mathrm{~m}, 24 \mathrm{H}), 0.88(\mathrm{~d}, 12 \mathrm{H}), 0.25(\mathrm{~s}, 9 \mathrm{H})$.

Synthesis of 5. A solution of 4 (4.08 mmol, $2.72 \mathrm{~g})$ in THF (240 $\mathrm{mL}$ ) was brought under argon atmosphere and shielded from light. Next, a tetrabutylammonium fluoride solution $(1.00 \mathrm{M}$, $12.6 \mathrm{~mL}$ ) in THF was added, and the reaction was stirred at room temperature. After $2 \mathrm{~h}$, diethyl ether $(100 \mathrm{~mL})$ and water $(100 \mathrm{~mL})$ were added. The aqueous layer was extracted with diethyl ether, and the collected organic fractions were washed with brine. Afterward, the solution was dried over $\mathrm{MgSO}_{4}$ and the solvents were removed under reduced pressure. Purification by column chromatography (eluent: dichloromethane) yielded a light red oil. Yield: $2.23 \mathrm{~g}(92 \%) .{ }^{1} \mathrm{H}$ NMR $\left(\mathrm{CDCl}_{3}\right): \delta=7.34(\mathrm{~s}$, $3 \mathrm{H}), 7.29$ (s, $1 \mathrm{H}), 4.52$ (s, $2 \mathrm{H}), 3.37$ (s, $1 \mathrm{H}), 2.82-2.70(\mathrm{~m}, 8 \mathrm{H})$,
1.67-1.65 (m, $8 \mathrm{H}), 1.34-1.23(\mathrm{~m}, 24 \mathrm{H}), 0.88(\mathrm{~d}, 12 \mathrm{H}) .{ }^{13} \mathrm{C}$ NMR $\left(\mathrm{CDCl}_{3}\right): \delta=143.3,142.8,142.3,132.9,132.8,123.4$, $122.9,122.5,104.3,99.5,93.3,92.6,84.5,54.5,54.1,53.7,53.4$, 53.0, 51.9, 34.5, 34.3, 32.3, 32.2, 31.1, 30.9, 29.7, 29.6, 23.1, 14.4. IR: $v\left(\mathrm{~cm}^{-1}\right)=3404,2954,2920,2852,1496,1457,860,836$. MS: $m / z=592\left(\mathrm{M}^{+}\right)$.

Synthesis of 6. A suspension of $\mathrm{MnO}_{2}(59.7 \mathrm{mmol}, 5.19 \mathrm{~g})$ and $\mathrm{KOH}(29.3 \mathrm{mmol}, 1.64 \mathrm{~g})$ in diethyl ether $(60 \mathrm{~mL})$ was brought under argon atmosphere and shielded from light. A solution of $\mathbf{5}$ $(3.76 \mathrm{mmol}, 2.23 \mathrm{~g})$ in diethyl ether $(10 \mathrm{~mL})$ was added, and the reaction was monitored by TLC. After completion, the suspension was filtered and the organic layer was washed with brine and dried over $\mathrm{MgSO}_{4}$. The crude product was purified by column chromatography (eluent: dichloromethane), yielding a red oil. Yield: $1.31 \mathrm{~g}(61 \%) .{ }^{1} \mathrm{H}$ NMR $\left(\mathrm{CD}_{2} \mathrm{Cl}_{2}\right): \delta=7.35(\mathrm{~s}, 4$ $\mathrm{H}), 3.36(\mathrm{~s}, 2 \mathrm{H}), 2.84-2.74(\mathrm{~m}, 8 \mathrm{H}), 1.68-1.66(\mathrm{~m}, 8 \mathrm{H})$, $1.35-1.28(\mathrm{~m}, 24 \mathrm{H}), 0.89(\mathrm{~d}, 12 \mathrm{H}) .{ }^{13} \mathrm{C} \mathrm{NMR}\left(\mathrm{CDCl}_{3}\right): \delta=$ 143.6, 142.7, 133.7, 133.1, 124.0, 122.2, 93.5, 83.0, 82.2, 34.8, 34.6, 32.6, 32.4 31.5, 31.3, 30.0, 29.8, 23.4, 14.6. IR: $v\left(\mathrm{~cm}^{-1}\right)=$ 2953, 2920, 2852, 1500, 1460, 865, 839. MS: $m / z=958(((\mathrm{M}-$ $\left.\left.\mathrm{C}_{6} \mathrm{H}_{13}\right)_{2}+\mathrm{Na}\right)^{+}$).

Synthesis of 7 . The same procedure as described for the synthesis of 2 was used, starting from 1 (4.75 mmol, $2.22 \mathrm{~g}$ ). The pure product was purified by column chromatography (eluent: $n$-hexane) and isolated as a gray solid. Yield: $2.00 \mathrm{~g}$ (85\%). mp: $104.5^{\circ} \mathrm{C} .{ }^{1} \mathrm{H} \mathrm{NMR}\left(\mathrm{CD}_{2} \mathrm{Cl}_{2}\right): \delta=7.39$ (s, $\left.4 \mathrm{H}\right), 7.34$ $(\mathrm{s}, 2 \mathrm{H}), 7.30(\mathrm{~s}, 2 \mathrm{H}), 2.84-2.71(\mathrm{~m}, 16 \mathrm{H}), 1.71-1.67(\mathrm{~m}, 16 \mathrm{H})$, $1.51-1.27(\mathrm{~m}, 48 \mathrm{H}), 0.88(\mathrm{~d}, 24 \mathrm{H}), 0.27(\mathrm{~s}, 18 \mathrm{H}) .{ }^{13} \mathrm{C} \mathrm{NMR}$ $\left(\mathrm{CD}_{2} \mathrm{Cl}_{2}\right): \delta=142.9,142.1,141.9,132.5,123.1,122.9,122.5$, 104.1, 99.1, 93.1, 34.3, 31.9, 31.8, 30.8, 29.4, 22.8, 14.3, 0.14. IR: $v\left(\mathrm{~cm}^{-1}\right)=2955,2919,2852,2149,1499,1458,839$. MS: $m / z=$ $1215\left(\left(\left(\mathrm{M}-\mathrm{C}_{45} \mathrm{H}_{65}\right)_{2}+\mathrm{Na}\right)^{+}\right)$.

Synthesis of $\mathbf{8}$. The same procedure as described for the synthesis of 5 was used, starting from 7 (1.20 mmol, $1.49 \mathrm{~g})$. A gray solid was obtained after the purification of the crude product by crystallization from cold methanol. Yield: $1.16 \mathrm{~g}$ (88\%). mp: $68{ }^{\circ} \mathrm{C} .{ }^{1} \mathrm{H}$ NMR $\left(\mathrm{CD}_{2} \mathrm{Cl}_{2}\right): \delta=7.38(\mathrm{~s}, 4 \mathrm{H}), 7.35(\mathrm{~s}$, $2 \mathrm{H}), 7.34$ (s, $2 \mathrm{H}), 3.36$ (s, $2 \mathrm{H}), 2.83-2.72(\mathrm{~m}, 16 \mathrm{H}), 1.70-1.60$ $(\mathrm{m}, 16 \mathrm{H}), 1.45-1.24(\mathrm{~m}, 48 \mathrm{H}), 0.88(\mathrm{~d}, 24 \mathrm{H}) .{ }^{13} \mathrm{C}$ NMR $\left(\mathrm{CD}_{2} \mathrm{Cl}_{2}\right): \delta=142.9,142.1,141.9,133.2,132.6,132.5,123.5$, 123.4, 123.3, 122.9, 122.8, 121.5, 93.2, 92.9, 82.6, 81.6, 34.4, 34.3, 32.0, 31.8, 30.8, 30.6, 29.4, 29.3, 22.8, 14.2. IR: $v\left(\mathrm{~cm}^{-1}\right)=2953$, 2919, 2851, 1500, 1457, 892. MS: $m / z=1099.8\left(\mathrm{M}^{+}\right)$.

Synthesis of 10. A suspension of $\mathrm{NaH}(55.0 \mathrm{mmol}, 1.32 \mathrm{~g})$ in dry DMF $(50.0 \mathrm{~mL})$ was purged with argon, and a solution of 9 $(25.0 \mathrm{mmol}, 9.05 \mathrm{~g})$ in dry DMF $(50.0 \mathrm{~mL})$ was dropwise added. The reaction mixture was stirred under argon at room temperature for $30 \mathrm{~min}$. Then, a solution of methyl bromoacetate $(60.0$ mmol, $9.18 \mathrm{~g})$ in DMF $(10.0 \mathrm{~mL})$ was added and the reaction mixture was stirred overnight at $40{ }^{\circ} \mathrm{C}$. Afterward, water $(10$ $\mathrm{mL}$ ) was added and a white precipitate was formed which was filtered off. The precipitate was thoroughly washed with water and diethyl ether, dried under vacuum, and isolated as a white solid. Yield: $8.10 \mathrm{~g}(64 \%)$. mp: $195.8^{\circ} \mathrm{C} .{ }^{1} \mathrm{H}$ NMR (DMSO-d6): $\delta=7.36(\mathrm{~s}, 2 \mathrm{H}), 4.91(\mathrm{~s}, 4 \mathrm{H}), 3.74(\mathrm{~s}, 6 \mathrm{H}),{ }^{13} \mathrm{C} \mathrm{NMR}\left(\mathrm{CDCl}_{3}\right)$ : $\delta=169.8,152.8,123.5,87.3,67.1,52.8$. IR: $v\left(\mathrm{~cm}^{-1}\right)=2962$, 2906, 1711, 1482, 1442, 1423, 1079, 848, 818. MS: $m / z=1035$ $\left((2 \mathrm{M}+\mathrm{Na})^{+}\right)$.

Synthesis of 11. A suspension of $\mathrm{NaH}(55.0 \mathrm{mmol}, 1.32 \mathrm{~g})$ in dry DMF $(50 \mathrm{~mL})$ was purged with argon, and a solution of 9 $(25.0 \mathrm{mmol}, 9.05 \mathrm{~g})$ in dry DMF $(50 \mathrm{~mL})$ was dropwise added. The reaction mixture was stirred at room temperature for 30 min. Then, a solution of methyl 6-bromohexanoate $(60.0 \mathrm{mmol}$, $12.5 \mathrm{~g})$ in dry DMF $(10 \mathrm{~mL})$ was added, and the reaction was stirred overnight at $40^{\circ} \mathrm{C}$. After reaction, dichloromethane $(20$ $\mathrm{mL}$ ) was added and the organic layer was washed respectively with a saturated $\mathrm{NaCl}$ solution and water. The organic phase was dried over $\mathrm{MgSO}_{4}$ and concentrated. The crude product was purified by column chromatography (eluent: $n$-hexane/ ethyl acetate $5 / 5$ ) and recrystallized from $n$-hexane to remove 
residual traces of methyl 6-bromohexanoate. The pure product was isolated as a white crystalline powder. Yield: $13.91 \mathrm{~g}(90 \%)$. mp: $96.0{ }^{\circ} \mathrm{C} .{ }^{1} \mathrm{H} \mathrm{NMR}\left(\mathrm{CDCl}_{3}\right): \delta=7.17(\mathrm{~s}, 2 \mathrm{H}), 3.94(\mathrm{t}, 4 \mathrm{H}), 3.68$ $(\mathrm{s}, 6 \mathrm{H}), 2.37(\mathrm{t}, 4 \mathrm{H}), 1.82(\mathrm{~m}, 4 \mathrm{H}), 1.70(\mathrm{~m}, 4 \mathrm{H}), 1.53(\mathrm{~m}, 4 \mathrm{H}) .{ }^{13} \mathrm{C}$ NMR $\left(\mathrm{CDCl}_{3}\right): \delta=174.4,153.1,123.1,86.6,70.3,51.9,34.3,29.1$, 26.0, 24.9. IR: $v\left(\mathrm{~cm}^{-1}\right)=2937,2875,1719,1488,1456,1421,1066$, 859. MS: $m / z=641\left((\mathrm{M}+\mathrm{Na})^{+}\right)$.

Synthesis of 12. $10(20.0 \mathrm{mmol}, 10.1 \mathrm{~g})$ and $\mathrm{Pd}\left(\mathrm{PPh}_{3}\right)_{2} \mathrm{Cl}_{2}$ $(0.150 \mathrm{mmol}, 105 \mathrm{mg})$ were dissolved in a mixture of dry ethyl acetate $(50 \mathrm{~mL})$ and dry triethylamine $(12.5 \mathrm{~mL})$ and purged with argon. The reaction mixture was heated at $40{ }^{\circ} \mathrm{C}$, and trimethylsilyl acetylene (46.0 mmol, $4.52 \mathrm{~g})$ was added. Next, $\mathrm{CuI}(4.00 \mathrm{mmol}, 762 \mathrm{mg})$ dissolved in a mixture of dry ethyl acetate $(8 \mathrm{~mL})$ and dry triethylamine $(2 \mathrm{~mL})$ was added, and the reaction mixture was stirred for $15 \mathrm{~min}$ at $40{ }^{\circ} \mathrm{C}$. Afterward, dichloromethane $(20 \mathrm{~mL})$ was added and the organic phase was washed with a $\mathrm{HCl}$ solution $(0.05 \mathrm{M})$, a saturated $\mathrm{NaCl}$ solution, and water, respectively. The organic phase was isolated and dried over $\mathrm{MgSO}_{4}$, and after concentration in vacuo, the crude product was purified by column chromatography (eluent: $n$-hexane/ethyl acetate $8 / 2$ ). The obtained product was isolated as a white solid. Yield: $4.32 \mathrm{~g}(48 \%)$. mp: $162.0{ }^{\circ} \mathrm{C}^{1} \mathrm{H}$ NMR $\left(\mathrm{CD}_{2} \mathrm{Cl}_{2}\right): \delta=6.86(\mathrm{~s}, 2 \mathrm{H}), 4.65(\mathrm{~s}, 4 \mathrm{H})$, $3.78(\mathrm{~s}, 6 \mathrm{H}), 0.24(\mathrm{~s}, 18 \mathrm{H}) .{ }^{13} \mathrm{C} \mathrm{NMR}\left(\mathrm{CDCl}_{3}\right): \delta=169.3,153.9$, 119.5, 115.2, 102.0, 100.2, 67.4, 52.6, 0.19. IR: $v\left(\mathrm{~cm}^{-1}\right)=2955$, 2913, 2157, 1769, 1530, 1437, 1407, 1089, 883. MS: $m / z=916$ $\left((2 \mathrm{M}+\mathrm{Na})^{+}\right)$.

Synthesis of $13.12(9.96 \mathrm{mmol}, 4.33 \mathrm{~g})$ was dissolved in THF $(250 \mathrm{~mL})$, brought under inert atmosphere, and protected from ambient light. The mixture was ice-cooled, and a solution of tetrabutylammonium fluoride trihydrate $(12.5 \mathrm{mmol}, 3.97 \mathrm{~g})$ in THF $(50 \mathrm{~mL})$ was slowly added. The reaction mixture was stirred for $5 \mathrm{~min}$ at $0^{\circ} \mathrm{C}$, followed by addition of water $(250 \mathrm{~mL})$ and ethyl acetate $(150 \mathrm{~mL})$. The organic phase was isolated and concentrated by rotatory evaporation, and the crude product was purified by recrystallization from a $n$-hexane/ethyl acetate mixture $(8 / 2)$. The pure product was filtered off, dried under vacuum, and isolated as a white solid. Yield: $1.77 \mathrm{~g}(60 \%)$. mp: $162.4{ }^{\circ} \mathrm{C} .{ }^{1} \mathrm{H}$ NMR $\left(\mathrm{CDCl}_{3}\right)$ : $\delta=7.03(\mathrm{~s}, 2 \mathrm{H}), 4.88(\mathrm{~s}, 4 \mathrm{H}), 4.44(\mathrm{~s}, 2 \mathrm{H}), 3.70(\mathrm{~s}, 6 \mathrm{H}) .{ }^{13} \mathrm{C} \mathrm{NMR}$ $\left(\mathrm{CDCl}_{3}\right): \delta=169.9,153.6,118.2,113.4,87.7,80.3,66.2$, 52.8. IR: $v\left(\mathrm{~cm}^{-1}\right)=2954,2911,1761,1500,1404,1084,851 . \mathrm{MS}: \mathrm{m} / z=$ $303\left(\mathrm{M}^{+}\right)$.

Synthesis of 14. The same procedure as described for the synthesis of $\mathbf{1 2}$ was used, starting from $\mathbf{1 0}(20.0 \mathrm{mmol}, 12.4 \mathrm{~g})$. Pure product was obtained after column chromatography (eluent: $n$-hexane/ethyl acetate $7 / 3$ ) and isolated as a white solid. Yield: $7.95 \mathrm{~g}(71 \%) \mathrm{mp}: 95.5^{\circ} \mathrm{C} .{ }^{1} \mathrm{H} N M R\left(\mathrm{CDCl}_{3}\right): \delta=6.88(\mathrm{~s}$, $2 \mathrm{H}), 3.95(\mathrm{t}, 4 \mathrm{H}), 3.67(\mathrm{~s}, 6 \mathrm{H}), 1.81(\mathrm{~m}, 4 \mathrm{H}), 1.71(\mathrm{~m}, 4 \mathrm{H}), 1.52$ $(\mathrm{m}, 4 \mathrm{H}), 0.25(\mathrm{~s}, 18 \mathrm{H}) \cdot{ }^{13} \mathrm{C} \mathrm{NMR}\left(\mathrm{CDCl}_{3}\right): \delta=174.4,154.2$, $117.5,114.2,101.2,100.6,69.3,51.9,34.4,29.3,25.9,25.0,0.34$. IR: $v\left(\mathrm{~cm}^{-1}\right)=2942,2902,2156,1739,1495,1467,1035,868$. MS: $m / z=582\left((\mathrm{M}+\mathrm{Na})^{+}\right)$.

Synthesis of 15. The same procedure as described for the synthesis of 13 was used, starting from 14 (14.2 mmol, $7.95 \mathrm{~g})$. Pure product was obtained after column chromatography (eluent: $n$-hexane/ethyl acetate 65/35) and isolated as a white solid. Yield: $4.31 \mathrm{~g}(73 \%)$. mp: $92.7^{\circ} \mathrm{C} .{ }^{1} \mathrm{H} \mathrm{NMR}\left(\mathrm{CDCl}_{3}\right): \delta=$ $6.94(\mathrm{~s}, 2 \mathrm{H}), 3.97(\mathrm{t}, 4 \mathrm{H}), 3.67(\mathrm{~s}, 6 \mathrm{H}), 2.34(\mathrm{~s}, 2 \mathrm{H}), 2.35(\mathrm{t}, 4 \mathrm{H})$, $1.82(\mathrm{~m}, 4 \mathrm{H}), 1.74(\mathrm{~m}, 4 \mathrm{H}), 1.52(\mathrm{~m}, 4 \mathrm{H}) .{ }^{13} \mathrm{C} \mathrm{NMR}\left(\mathrm{CDCl}_{3}\right)$ : $\delta=174.4,154.2,118.0,113.6,82.9,79.9,69.6,51.9,34.3,29.1$, 25.9, 24.9. IR: $v\left(\mathrm{~cm}^{-1}\right)=2947,2869,1720,1499,1467,1436$, 1039, 862. MS: $m / z=438\left((\mathrm{M}+\mathrm{Na})^{+}\right)$.

Synthesis of 16. $13(4.50 \mathrm{mmol}, 1.36 \mathrm{~g})$ and $\mathrm{Pd}\left(\mathrm{PPh}_{3}\right)_{2} \mathrm{Cl}_{2}$ $(0.225 \mathrm{mmol}, 158 \mathrm{mg})$ were dissolved in a mixture of ethyl acetate $(100 \mathrm{~mL})$ and triethylamine $(25 \mathrm{~mL})$ and purged with argon. The mixture was heated at $60{ }^{\circ} \mathrm{C}$, followed by the addition of 1 (9.10 mmol, $4.26 \mathrm{~g})$ and $\mathrm{CuI}(0.90 \mathrm{mmol}, 0.171$ $\mathrm{g})$, both dissolved in a mixture of ethyl acetate $(8 \mathrm{~mL})$ and triethylamine $(2 \mathrm{~mL})$. The reaction mixture was stirred for $30 \mathrm{~min}$, and afterward, water $(20 \mathrm{~mL})$ and dichloromethane $(50 \mathrm{~mL})$ were added. The organic phase was washed with a diluted $\mathrm{HCl}$ solution $(0.05 \mathrm{M})$, saturated $\mathrm{NaCl}$ solution, and water, respectively. The organic layer was dried over $\mathrm{MgSO}_{4}$ and concentrated in vacuo. The crude product was purified by column chromatography (eluent: $n$-hexane/ethyl acetate 9/1) and isolated as a yellow solid. Yield: $0.86 \mathrm{~g}(20 \%)$. mp: $83.6{ }^{\circ} \mathrm{C} .{ }^{1} \mathrm{H}$ NMR $\left(\mathrm{CDCl}_{3}\right): \delta=7.34(\mathrm{~s}, 2 \mathrm{H}), 7.29(\mathrm{~s}, 2 \mathrm{H}), 6.96(\mathrm{~s}, 2 \mathrm{H}), 4.72(\mathrm{~s}, 4 \mathrm{H})$, $3.81(\mathrm{~s}, 6 \mathrm{H}), 2.79(\mathrm{t}, 4 \mathrm{H}), 2.72(\mathrm{t}, 4 \mathrm{H}), 1.63(\mathrm{~m}, 8 \mathrm{H}), 1.31(\mathrm{~m}, 24 \mathrm{H})$, $0.88(\mathrm{~m}, 12 \mathrm{H}), 0.26(\mathrm{~s}, 18 \mathrm{H}) \cdot{ }^{13} \mathrm{C} \mathrm{NMR}\left(\mathrm{CDCl}_{3}\right): \delta=169.3,153.5$, $143.1,142.6,132.8,132.7,123.0,122.8,118.2,115.2,104.3,99.4$, $95.3,89.9,67.2,52.6,34.5,34.3,32.1,30.9,29.6,29.5,22.9,14.4$, 0.32. IR: $v\left(\mathrm{~cm}^{-1}\right)=2954,2923,2853,2145,1766,1504,1417,1087$, 838. MS: $m / z=1989\left((2 \mathrm{M}+\mathrm{Na})^{+}\right)$.

Synthesis of 17. The same procedure as described for the synthesis of 13 was used, starting from $16(0.876 \mathrm{mmol}, 861 \mathrm{mg})$. Pure product was obtained after column chromatography (eluent: $n$-hexane/ethyl acetate 9/1) and isolated as a brown oil. Yield: $0.50 \mathrm{~g}(69 \%)$. ${ }^{1} \mathrm{H}$ NMR $\left(\mathrm{CDCl}_{3}\right): \delta=7.37(\mathrm{~s}, 2 \mathrm{H})$, $7.32(\mathrm{~s}, 2 \mathrm{H}), 6.97(\mathrm{~s}, 2 \mathrm{H}), 4.73(\mathrm{~s}, 4 \mathrm{H}), 3.81(\mathrm{~s}, 6 \mathrm{H}), 3.31(\mathrm{~s}, 2 \mathrm{H})$, $2.81(\mathrm{t}, 4 \mathrm{H}), 2.74(\mathrm{t}, 4 \mathrm{H}), 1.66(\mathrm{~m}, 8 \mathrm{H}), 1.31(\mathrm{~m}, 24 \mathrm{H}), 0.88(\mathrm{~m}$, $12 \mathrm{H}) .{ }^{13} \mathrm{C} \mathrm{NMR}\left(\mathrm{CDCl}_{3}\right): \delta=169.0,153.3,142.8,142.4,133.1$, 132.5, 123.0, 121.9, 117.8, 114.9, 95.0, 89.8, 82.6, 81.9, 66.8, 66.0, 52.24, 34.1, 34.0, 32.1, 31.9, 30.7, 29.9, 29.6, 29.4, 22.8, 15.4, 14.2. MS: $m / z=862\left((\mathrm{M}+\mathrm{Na})^{+}\right)$.

Synthesis of 18. The same procedure as described for the synthesis of 16 was used, starting from 15 (5.00 mmol, $2.07 \mathrm{~g})$. Pure product was obtained after column chromatography (eluent: $n$-hexane/ethyl acetate 9/1) and isolated as a yellow solid. Yield: $3.00 \mathrm{~g}(55 \%)$. mp: $78.3{ }^{\circ} \mathrm{C} .{ }^{1} \mathrm{H} \mathrm{NMR}\left(\mathrm{CDCl}_{3}\right): \delta=$ $7.31(\mathrm{~s}, 2 \mathrm{H}), 7.29(\mathrm{~s}, 2 \mathrm{H}), 6.96(\mathrm{~s}, 2 \mathrm{H}), 4.02(\mathrm{t}, 4 \mathrm{H}), 3.64(\mathrm{~s}, 6 \mathrm{H})$, $2.80(\mathrm{t}, 4 \mathrm{H}), 2.72(\mathrm{t}, 4 \mathrm{H}), 2.32(\mathrm{t}, 4 \mathrm{H}), 1.87(\mathrm{~m}, 4 \mathrm{H}), 1.67(\mathrm{~m}$, $12 \mathrm{H}), 1.31(\mathrm{~m}, 28 \mathrm{H}), 0.87(\mathrm{~m}, 12 \mathrm{H}), 0.26(\mathrm{~s}, 18 \mathrm{H}) .{ }^{13} \mathrm{C} \mathrm{NMR}$ $\left(\mathrm{CDCl}_{3}\right): \delta=174.3,153.7,143.0,142.3,132.8,132.6,123.2$, 122.7, 116.9, 114.4, 104.4, 99.3, 94.3, 90.8, 69.4, 51.8, 34.5, 34.3, $32.1,30.9,29.6,29.5,29.4,25.9,25.0,22.9,14.4,0.33$. IR: $v$ $\left(\mathrm{cm}^{-1}\right)=2950,2924,2855,2147,1737,1506,1433,1026,838$. MS: $m / z=1120\left((\mathrm{M}+\mathrm{Na})^{+}\right)$.

Synthesis of 19. The same procedure as described for the synthesis of 17 was used, starting from $18(5.00 \mathrm{mmol}, 2.07 \mathrm{~g})$. Pure product was obtained after column chromatography (eluent: $n$ hexane/ethyl acetate 9/1) and isolated as a yellow solid. Yield: $1.60 \mathrm{~g}$ (74\%). mp: $91.3{ }^{\circ} \mathrm{C} .{ }^{1} \mathrm{H}$ NMR $\left(\mathrm{CDCl}_{3}\right): \delta=7.34$ (s, $\left.2 \mathrm{H}\right), 7.33(\mathrm{~s}$, $2 \mathrm{H}), 6.98(\mathrm{~s}, 2 \mathrm{H}), 4.03(\mathrm{t}, 4 \mathrm{H}), 3.64(\mathrm{~s}, 6 \mathrm{H}), 3.30(\mathrm{~s}, 2 \mathrm{H}), 2.81(\mathrm{t}, 4 \mathrm{H})$, $2.74(\mathrm{t}, 4 \mathrm{H}), 2.32(\mathrm{t}, 4 \mathrm{H}), 1.87(\mathrm{~m}, 4 \mathrm{H}), 1.69(\mathrm{~m}, 12 \mathrm{H}), 1.31(\mathrm{~m}$, $28 \mathrm{H}), 0.88(\mathrm{~m}, 12 \mathrm{H}) .{ }^{13} \mathrm{C} \mathrm{NMR}\left(\mathrm{CDCl}_{3}\right): \delta=174.3,153.7,143.1$, 142.4, 133.3, 132.6, 123.6, 121.7, 116.9, 114.4, 94.1, 90.8, 82.8, 81.8, $69.4,51.8,34.3,34.2,34.1,32.1,32.0,30.8,30.0,29.4,29.3,25.9$, 25.0, 22.9, 14.4. IR: $v\left(\mathrm{~cm}^{-1}\right)=2949,2925,2850,1726,1504,1485$, 1457, 1014, 844. MS: $m / z=1925\left((2 \mathrm{M}+\mathrm{Na})^{+}\right)$.

General Synthesis of P1-4. A solution of $22(0.800 \mathrm{mmol}$, $0.509 \mathrm{~g})$ and $\mathrm{Pd}\left(\mathrm{PPh}_{3}\right)_{4}(0.0160 \mathrm{mmol}, 18.5 \mathrm{mg})$ in a mixture of piperidine $(1.5 \mathrm{~mL})$ and $\mathrm{THF}(3.5 \mathrm{~mL})$ was purged with argon. The reaction mixture was heated at $60{ }^{\circ} \mathrm{C}$, and $\mathbf{2 4}(0.800 \mathrm{mmol}$, $0.236 \mathrm{~g})$, dissolved in THF $(0.5 \mathrm{~mL})$, and $\mathrm{CuI}(36.0 \mu \mathrm{mol}, 63.9$ $\mathrm{mg})$, dissolved in a mixture of piperidine/THF $(3 / 7,0.5 \mathrm{~mL})$, were added respectively. The reaction mixture was stirred overnight, and afterward, $\mathrm{CHCl}_{3}$ was added. The organic layer was washed with a $\mathrm{HCl}$ solution $(0.500 \mathrm{M})$ and water and dried with $\mathrm{Na}_{2} \mathrm{SO}_{4}$. After concentration by rotatory evaporation, the polymer solution was precipitated in methanol, filtered off, and dried. Further purification was accomplished by Soxhlet extractions with methanol, $n$-pentane, $n$-hexane, and $\mathrm{CHCl}_{3}$, respectively. The chloroform-soluble fraction was concentrated and precipitated in methanol. Finally, the polymer was filtered off and dried. P1: Yield $=499 \mathrm{mg}(81 \%)$. ${ }^{1} \mathrm{H}$ NMR $\left(\mathrm{CD}_{2} \mathrm{Cl}_{2}\right)$ : $\delta=7.34(\mathrm{~s}, 2 \mathrm{H}), 7.05(\mathrm{~s}, 2 \mathrm{H}), 4.20(\mathrm{~s}, 4 \mathrm{H}), 2.78(\mathrm{~s}, 4 \mathrm{H}), 1.94-1.69$ $(\mathrm{m}, 8 \mathrm{H}), 1.51-1.19(\mathrm{~m}, 28 \mathrm{H}), 0.85(\mathrm{~m}, 24 \mathrm{H}) . \mathrm{IR}: v\left(\mathrm{~cm}^{-1}\right)=2951$, 2922, 2855, 2187, 1525, 1457, 1409, 1060, 806. P2: Yield $=536 \mathrm{mg}$ $(72 \%) .{ }^{1} \mathrm{H}$ NMR $\left(\mathrm{CD}_{2} \mathrm{Cl}_{2}\right): \delta=7.36(\mathrm{~s}, 4 \mathrm{H}), 7.05(\mathrm{~s}, 2 \mathrm{H}), 4.20(\mathrm{~s}$, $4 \mathrm{H}), 2.83(\mathrm{~s}, 8 \mathrm{H}), 1.94-1.69(\mathrm{~m}, 8 \mathrm{H}), 1.51-1.19(\mathrm{~m}, 44 \mathrm{H}), 0.86(\mathrm{~m}$, 30H). IR: $v\left(\mathrm{~cm}^{-1}\right)=2952,2922,2854,2184,1524,1456,1411,893$, 
Scheme 1. Synthesis of Monomers 6 and $8^{a}$
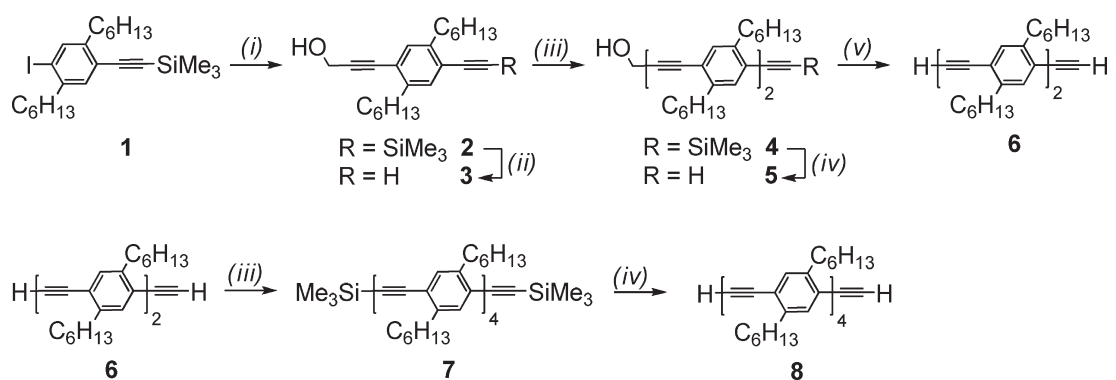

a (i) propargyl alcohol, $\mathrm{Pd}\left(\mathrm{PPh}_{3}\right)_{2} \mathrm{Cl}_{2}, \mathrm{CuI}, \mathrm{THF} /{ }^{i} \mathrm{Pr}_{2} \mathrm{NH}$; (ii) $\mathrm{NaOH}$ (aq), THF; (iii) 1, $\mathrm{Pd}\left(\mathrm{PPh}_{3}\right)_{2} \mathrm{Cl}_{2}, \mathrm{CuI}, \mathrm{THF} / \mathrm{Pr}_{2} \mathrm{NH}$; (iv) TBAF, THF; (v) MnO , $\mathrm{KOH}, \mathrm{Et}_{2} \mathrm{O}$.

Scheme 2. Synthesis of Monomers 17 and $19^{a}$

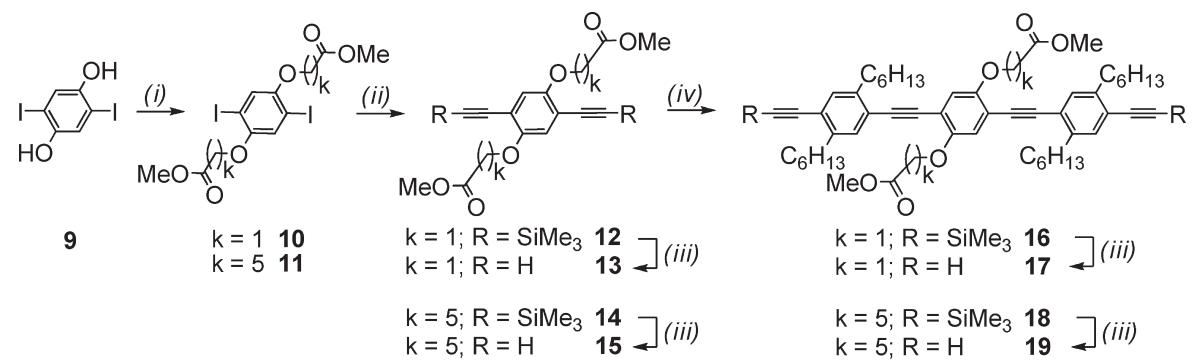

${ }^{a}($ i $) \mathrm{NaH}$, methyl bromoacetate (10), or methyl 6-bromohexanoate (11); (ii) trimethylsilylacetylene, $\left.\mathrm{Pd}_{(} \mathrm{PPh}_{3}\right)_{2} \mathrm{Cl}_{2}, \mathrm{CuI}, \mathrm{Et}_{3} \mathrm{~N} / \mathrm{ethyl}$ acetate; (iii) TBAF, THF; (iv) 1, $\mathrm{Pd}\left(\mathrm{PPh}_{3}\right)_{2} \mathrm{Cl}_{2}, \mathrm{CuI}, \mathrm{Et}_{3} \mathrm{~N} / \mathrm{EtOAc}$.

807. P3: Yield $=165 \mathrm{mg}(79 \%) .{ }^{1} \mathrm{H}$ NMR $\left(\mathrm{CDCl}_{3}\right): \delta=7.38(\mathrm{~s}$, $6 \mathrm{H}), 7.05(\mathrm{~s}, 2 \mathrm{H}), 4.21(\mathrm{~s}, 4 \mathrm{H}), 2.83(\mathrm{~s}, 12 \mathrm{H}), 1.94-1.71(\mathrm{~m}, 8 \mathrm{H})$, $1.55-1.20(\mathrm{~m}, 60 \mathrm{H}), 0.87(\mathrm{~m}, 36 \mathrm{H})$. IR: $v\left(\mathrm{~cm}^{-1}\right)=2952,2922$, 2954, 2182, 1524, 1456, 1411, 894, 807. P4: Yield $=276 \mathrm{mg}(22 \%)$. ${ }^{1} \mathrm{H} \mathrm{NMR}\left(\mathrm{CDCl}_{3}\right): \delta=7.39(\mathrm{~s}, 8 \mathrm{H}), 7.05(\mathrm{~s}, 2 \mathrm{H}), 4.20(\mathrm{~s}, 4 \mathrm{H}), 2.83$ $(\mathrm{s}, 16 \mathrm{H}), 1.94-1.71(\mathrm{~m}, 8 \mathrm{H}), 1.35-1.18(\mathrm{~m}, 76 \mathrm{H}), 0.88(\mathrm{~m}, 42 \mathrm{H})$. IR: $v\left(\mathrm{~cm}^{-1}\right)=2953,2921,2853,2183,1525,1456,1411,893,805$.

General Synthesis of P5a-8a. Monomer $22(0.060 \mathrm{mmol}, 50.0$ $\mathrm{mg})$ and $\mathrm{Pd}\left(\mathrm{PPh}_{3}\right)_{4}(1.49 \mu \mathrm{mol}, 1.73 \mathrm{mg})$ were dissolved in a mixture of THF and triethylamine $(8 / 2,2 \mathrm{~mL})$, and the solution was purged with argon and protected from ambient light. The reaction mixture was heated at $40^{\circ} \mathrm{C}$ and a solution of monomer $17(0.060 \mathrm{mmol}, 37.9 \mathrm{mg})$ was added, followed by the addition of $\mathrm{CuI}(3.00 \mu \mathrm{mol}, 0.567 \mathrm{mg})$. The reaction mixture was stirred overnight, and afterward $\mathrm{CHCl}_{3}(2 \mathrm{~mL})$ was added. The organic phase was washed with a $\mathrm{HCl}$ solution $(0.05 \mathrm{M})$, a saturated $\mathrm{NaCl}$ solution, and water, respectively, and dried over $\mathrm{Na}_{2} \mathrm{SO}_{4}$. The polymer solution was concentrated and poured into methanol. The precipitate was filtered off, and further purification was accomplished by Soxhlet extractions with acetone, $n$-hexane, and $\mathrm{CHCl}_{3}$, respectively. The chloroform-soluble fraction was concentrated and precipitated in methanol. Finally, the polymer was filtered off and dried. P5a: Yield $=15 \mathrm{mg}(10 \%) .{ }^{1} \mathrm{H}$ NMR $\left(\mathrm{CDCl}_{3}\right): \delta=7.39(\mathrm{~s}, 2 \mathrm{H}), 7.33(\mathrm{~s}, 2 \mathrm{H}), 7.02(\mathrm{~s}, 2 \mathrm{H}), 6.98(\mathrm{~s}, 2 \mathrm{H})$, $4.74(\mathrm{~s}, 4 \mathrm{H}), 4.18(\mathrm{~s}, 4 \mathrm{H}), 3.83(\mathrm{~s}, 6 \mathrm{H}), 2.81(\mathrm{~s}, 8 \mathrm{H}), 1.95-1.36(\mathrm{~m}$, $54 \mathrm{H}), 0.88(\mathrm{~m}, 30 \mathrm{H})$. IR: $v\left(\mathrm{~cm}^{-1}\right)=2952,2923,2854,2186$, 1763, 1504, 1455, 1435, 1082, 854, 806. P6a: Yield $=34 \mathrm{mg}$ $(22 \%) .{ }^{1} \mathrm{H} \mathrm{NMR}\left(\mathrm{CDCl}_{3}\right): \delta=7.39(\mathrm{~s}, 2 \mathrm{H}), 7.34(\mathrm{~s}, 2 \mathrm{H}), 7.01(\mathrm{~s}$, $2 \mathrm{H}), 6.98(\mathrm{~s}, 2 \mathrm{H}), 4.74(\mathrm{~s}, 4 \mathrm{H}), 4.14(\mathrm{~s}, 4 \mathrm{H}), 3.83(\mathrm{~s}, 6 \mathrm{H}), 2.80(\mathrm{~s}$, $8 \mathrm{H}), 1.88-1.32(\mathrm{~m}, 32 \mathrm{H}), 0.88(\mathrm{~m}, 18 \mathrm{H})$. IR: $v\left(\mathrm{~cm}^{-1}\right)=2922$, 2853, 2182, 1765, 1525, 1454, 1412, 1063, 891. P7a: Yield $=64$ $\mathrm{mg}(43 \%) .{ }^{1} \mathrm{H}$ NMR $\left(\mathrm{CDCl}_{3}\right): \delta=7.36(\mathrm{~s}, 4 \mathrm{H}), 6.99(\mathrm{~s}, 4 \mathrm{H}), 4.17$ $(\mathrm{s}, 4 \mathrm{H}), 4.04(\mathrm{~s}, 4 \mathrm{H}), 3.65(\mathrm{~s}, 6 \mathrm{H}), 2.82(\mathrm{~s}, 8 \mathrm{H}), 2.34(\mathrm{~s}, 4 \mathrm{H})$, $1.80-1.21(\mathrm{~m}, 64 \mathrm{H}), 0.87(\mathrm{~m}, 30 \mathrm{H})$. IR: $v\left(\mathrm{~cm}^{-1}\right)=2924,2855$, 2342, 2183, 1738, 1456, 1418, 1063, 893. P8a: Yield $=109 \mathrm{mg}$ $(76 \%) .{ }^{1} \mathrm{H}$ NMR $\left(\mathrm{CDCl}_{3}\right): \delta=7.36(\mathrm{~s}, 4 \mathrm{H}), 6.99(\mathrm{~s}, 4 \mathrm{H}), 4.15(\mathrm{~s}$, $4 \mathrm{H}), 4.04(\mathrm{~s}, 4 \mathrm{H}), 3.65(\mathrm{~s}, 6 \mathrm{H}), 2.82(\mathrm{~s}, 8 \mathrm{H}), 2.34(\mathrm{~s}, 4 \mathrm{H})$, $1.89-1.25(\mathrm{~m}, 68 \mathrm{H}), 0.88(\mathrm{~m}, 18 \mathrm{H})$. IR: $v\left(\mathrm{~cm}^{-1}\right)=2922$, 2854, 2177, 1738, 1524, 1417, 1050, 894.
General Synthesis of $\mathbf{P} \boldsymbol{6} \boldsymbol{b}-\boldsymbol{8 b}$. Polymer 8a $(29.1 \mu \mathrm{mol}, 40.0 \mathrm{mg})$ was dissolved in THF $(25 \mathrm{~mL})$ and stirred under argon atmosphere. An aqueous $\mathrm{KOH}$ solution $(0.500 \mathrm{M}, 0.2 \mathrm{~mL})$ was added dropwise, and the mixture was stirred until complete conversion was reached (monitored by IR spectroscopy). Afterward, chloroform was added and the polymer solution was thoroughly washed with a $\mathrm{HCl}$ solution $(0.05 \mathrm{M})$ and water. The organic layer was isolated and dried over $\mathrm{Na}_{2} \mathrm{SO}_{4}$ and concentrated. Finally, the polymer was precipitated in ice-cooled methanol, filtered off, and dried. P6b: Yield $=16 \mathrm{mg}(53 \%)$. IR: $v\left(\mathrm{~cm}^{-1}\right)=2922,2853,2360,1714$, 1455, 1430, 1051, 886. P7b: Yield $=37 \mathrm{mg}(74 \%)$. IR: $v\left(\mathrm{~cm}^{-1}\right)=$ 2921, 2855, 2359, 2335, 1709, 1456, 1050, 859. P8b: Yield $=35 \mathrm{mg}$ $(70 \%)$. IR: $v\left(\mathrm{~cm}^{-1}\right)=2924,2855,2360,2341,1708,1414$, 1050,805 .

\section{Results and Discussion}

Monomer and Polymer Synthesis. The polymers were prepared by a Sonogashira reaction between a dibromo-functionalized bithiophene and an oligo(para-phenylene ethynlene) (OPE). The Sonogashira reaction also plays a crucial role in the synthetic strategy for OPEs. It has however been reported ${ }^{9}$ that a controlled elongation of OPEs is only possible by selective protection/deprotection steps of the acetylene moieties.

As already mentioned, both OPEs with and without ester functionalities were prepared. The synthesis of dimer 6 is depicted in Scheme 1 and starts from 1. After a Sonogashira reaction with propargyl alcohol, $\mathbf{2}$ is formed with two different protecting groups. The polarity change accompanying the transition of $\mathbf{1}$ to $\mathbf{2}$ allows an easy chromatographic purification. Selective deprotection with $\mathrm{NaOH}$ leads to $\mathbf{3}$, on which again a Sonogashira reaction is performed with $\mathbf{1}$, resulting in 4 . The trimethylsilyl group was removed by reaction of $\mathbf{4}$ with tetrabutylammonium fluoride (TBAF), affording $\mathbf{5}$. The use of TBAF results in a clean deprotection, while on the other hand deprotection with $\mathrm{NaOH}$ leads to side-products which complicate the purification. Dimer 6 was obtained after removal of the alcoholic protection 
group, which was accomplished with $\mathrm{NaOH}$ in the presence of $\mathrm{MnO}_{2}$. Tetramer $\mathbf{8}$, finally, was synthesized starting from 6 by performing a Sonogashira reaction with 2 equiv of $\mathbf{1}$. A double deprotection step on 7 with TBAF yielded 8 .

Scheme 2 displays the synthesis of the ester-functionalized trimers $\mathbf{1 7}$ and 19. Both trimers were synthesized based on a similar synthetic strategy. Starting from 9, deprotonation of the phenolic groups was accomplished with $\mathrm{NaH}$, which was followed by a nucleophilic substitution on methyl bromoacetate or methyl 6-bromoacetate, leading to $\mathbf{1 0}$ and $\mathbf{1 1}$, respectively. Again, a Sonogashira reaction with trimethylsilyl acetylene was performed, yielding 12 and 14. The trimethylsilyl groups were removed by means of TBAF. Next, both 13 and 15 underwent a Sonogashira reaction with 2 equiv of $\mathbf{1}$, resulting in $\mathbf{1 6}$ and $\mathbf{1 8}$, respectively. A final deprotection step with TBAF converted 16/18 into 17/19.

Scheme 3 shows the synthesis of $\mathbf{P 1 - 4}$. The polymerizations were carried out in a mixture of piperidine and THF $(3 / 7)$, in which the polymers are readily soluble. Removal of residual monomers and oligomers was accomplished by Soxhlet extraction with different solvents (acetone and $n$-hexane). The polymers were extracted with chloroform,

Scheme 3. Synthesis of Polymers P1-4

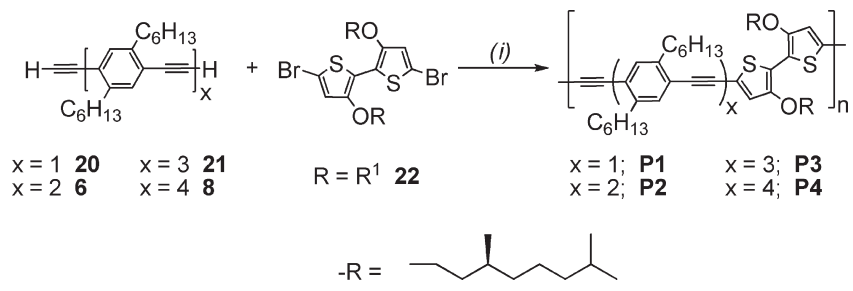

${ }^{a}\left(\right.$ i) $\mathrm{Pd}\left(\mathrm{PPh}_{3}\right)_{4}, \mathrm{CuI}$, piperidine/THF. precipitated in methanol, filtered off, and dried, giving P1-P4 in moderate to high yields.

The synthesis of the ester-functionalized polymers is displayed in Scheme 4 and is again based on a Sonogashira reaction. Using the chiral (22) and achiral (23) bithiophene monomers, the chiral polymers P5a/P7a and the achiral polymers P6a/P8a were obtained. The same purification procedure was performed as described for $\mathbf{P 1 - 4}$, resulting in P5a-P8a in low to moderate yields. The structure of all polymers (P1-4 and P5a-8a) was confirmed by ${ }^{1} \mathrm{H}$ NMR analysis.

The ester-functionalized polymers were saponificated with an aqueous potassium hydroxide solution (Scheme 5). The deprotection was performed in THF in the presence of small amounts of water and methanol. It appeared crucial to work in a diluted medium to avoid polymer degradation. The saponification was monitored by means of IR spectroscopy. A clear shift of the carbonyl stretch from $1740 \mathrm{~cm}^{-1}$ (ester) to $1710 \mathrm{~cm}^{-1}$ (carboxylic acid) was observed (Figure S1 of the Supporting Information). After complete carboxylate formation, chloroform was added, and the polymer solution was acidified with $\mathrm{HCl}$. The polymers $\mathbf{P 5} \mathbf{b}-\mathbf{8 b}$ were precipitated in methanol, filtered off, and dried.

GPC and DSC Analysis. P1-4 and P5a-P8a show an excellent solubility in common organic solvents, such as chloroform and THF. In contrast, $\mathbf{P 5 b}-\mathbf{P 8 b}$ are moderately soluble in THF, only poorly soluble in chloroform, and insoluble in most other solvents.

The molar mass (Table 1) was measured by GPC in THF toward polystyrene standards. All polymers, except P4, show polydispersities between 2 and 3, which are typical for polycondensation. The higher polydispersity of $\mathbf{P} 4$ probably originates from aggregation during polymerization.

DSC experiments were performed on $\mathbf{P 1}-\mathbf{4}$ and $\mathbf{P 5 a}-\mathbf{8 a}$, showing for all polymers but P5a a clear glass transition. In

Scheme 4. Synthesis of Polymers P5a-8a

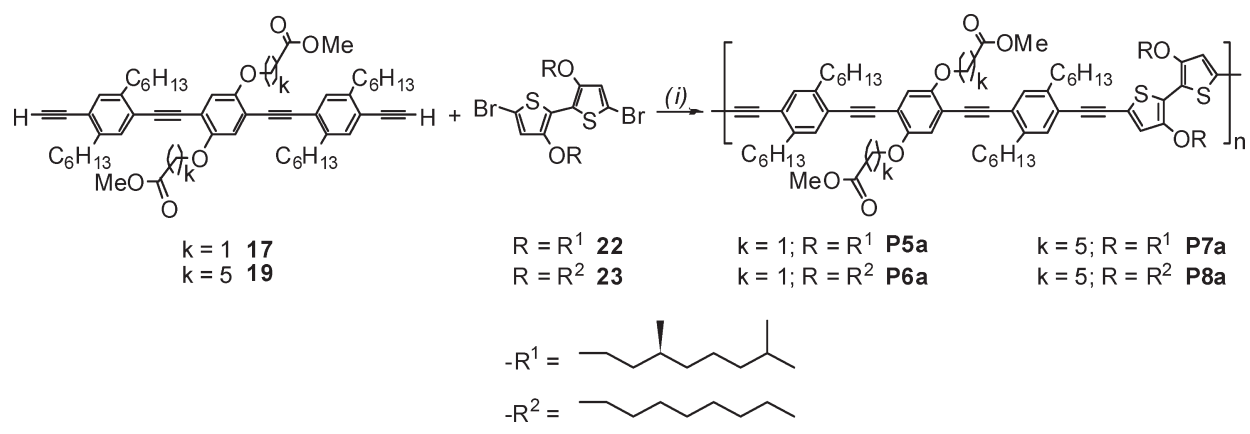

${ }^{a}(i) \mathrm{Pd}\left(\mathrm{PPh}_{3}\right)_{4}, \mathrm{CuI}, \mathrm{THF} / \mathrm{Et}_{3} \mathrm{~N}$.

Scheme 5. Synthesis of Polymers P5b $-8 b^{a}$

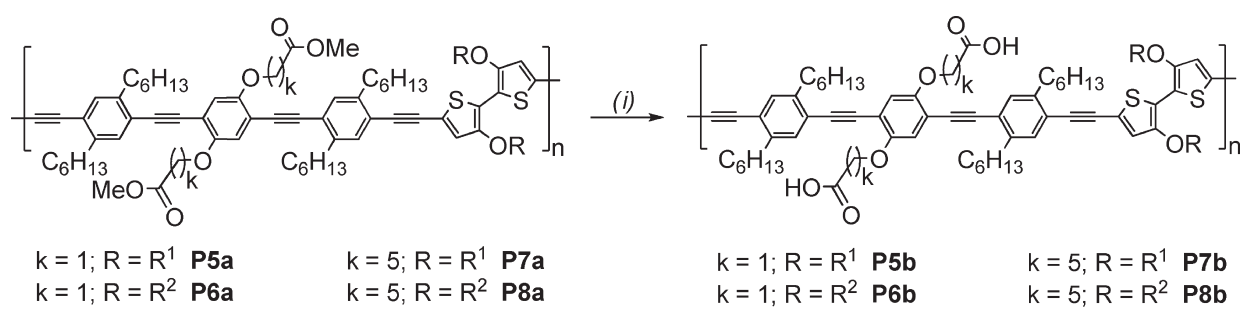

${ }^{a}(i) \mathrm{KOH}, \mathrm{THF} / \mathrm{MeOH} / \mathrm{H}_{2} \mathrm{O}$.

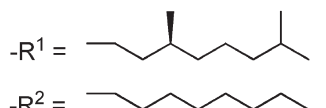


Table 1. Number Average Molar Mass $\left(\bar{M}_{\mathrm{n}}\right)$, Weight Average Molar Mass $\left(\bar{M}_{\mathrm{w}}\right)$, Polydispersity $(D)$, Degree of Polymerization $\left(\bar{X}_{\mathrm{n}}\right)$, Glass Transition Temperature $\left(T_{\mathrm{g}}\right)$, and Melting Temperature $\left(T_{\mathrm{m}}\right)$ of P1 -4 and $P 5 a-8 a$

\begin{tabular}{lcccccc}
\hline & $\begin{array}{c}\bar{M}_{\mathrm{n}} \\
\left(\mathrm{kg} \cdot \mathrm{mol}^{-1}\right)^{a}\end{array}$ & $\begin{array}{c}\bar{M}_{\mathrm{w}} \\
\left(\mathrm{kg} \cdot \mathrm{mol}^{-1}\right)^{a}\end{array}$ & $D^{a}$ & $\bar{X}_{n}$ & $T_{\mathrm{g}}\left({ }^{\circ} \mathrm{C}\right)^{b}$ & $T_{\mathrm{m}}\left({ }^{\circ} \mathrm{C}^{b}\right.$ \\
\hline P1 & 26.0 & 64.0 & 2.5 & 33 & 167 & 197 \\
P2 & 24.0 & 57.0 & 2.4 & 23 & 104 & 150 \\
P3 & 68.0 & 179 & 2.6 & 52 & 158 & 201 \\
P4 & 43.0 & 174 & 4.0 & 27 & 132 & 183 \\
P5a & 20.0 & 39.5 & 2.0 & 15 & & \\
P6a & 12.6 & 32.0 & 2.6 & 10 & 130 & \\
P7a & 18.5 & 38.1 & 2.1 & 13 & 148 & \\
P8a & 38.0 & 105 & 2.8 & 28 & 121 & \\
${ }^{a}$ Determined by GPC toward polystyrene & standards in & THF. \\
${ }^{b}$ Determined by DSC. Heating rate: $40{ }^{\circ} \mathrm{C} /$ min. & &
\end{tabular}

addition, P1-4 also show clear melting peaks, revealing their semicrystalline behavior. For the ester-functionalized polymers P5a-8a, no melting peaks could, however, be observed.

Chiroptical Properties: Solvatochromism. Polymers P1-4. In these polymers, the length of the achiral OPE was varied, while keeping the chiral bithiophene unit constant. Consequently, a comparison of the chiroptical behavior allows for the determination of the optimal length of the OPE spacer in terms of chiral expression, sensitivity toward external stimuli (e.g., nonsolvent), and synthetic accessibility. In Figure 2, the $\mathrm{UV}$ - vis and $\mathrm{CD}$ spectra in various $\mathrm{CHCl}_{3} / \mathrm{iPrOH}$ mixtures are displayed. $i \mathrm{PrOH}$ and not, for instance, methanol was used as nonsolvent, since precipitation of the polymers occurred in the latter solvent. Except for P4, decreasing the solvent quality results in a red-shift and the occurrence of a sharp band, which can be attributed to a planarization and stacking of the polymer chains. ${ }^{1 \mathrm{~b}} \lambda_{\max }$ of $\mathbf{P 1} \mathbf{- 4}$ is intermediate between that of the respective homopolymers $\left(\lambda_{\max }=580 / 610 \mathrm{~nm}\right.$ for HH-TT P3AOTs $^{6}$ and $400 / 438 \mathrm{~nm}$ for PPEs ${ }^{1 \mathrm{~b}}$ ) in both dissolved and aggregated states and decreases with increasing OPE length, which nicely reflects the different conjugation lengths of the bithiophene unit and the OPE parts. The solvatochromism is accompanied by the emergence of circular dichroism. More in particular, the CD spectra of $\mathbf{P 1 - 3}$ are composed of a bisignate Cotton effect in the $\pi-\pi^{*}$ transition with a zero-crossing around $450-480 \mathrm{~nm}$, originating from chiral exciton coupling of chirally stacked polymer chains, ${ }^{5 a}$ together with a monosignate Cotton effect (positive for P1 and P3, negative for P2) corresponding to the sharp, red-shifted band. ${ }^{\text {Sd,e }}$ The CD spectrum of P4 is unclear and much less intense. Probably, already some aggregation occurs in good solvent, which complicates the stacking. The poor solubility and presence of aggregates also explains its divergent $\mathrm{UV}-$ vis spectra.

A closer inspection of the UV-vis and CD spectra (Figure 2) reveals that the transition from unordered to chirally stacked polymer chains occurs within a rather small window of solvent composition. For instance, the major changes in the UV-vis spectra of $\mathbf{P} \mathbf{3}$ are observed from 41.9 to $50.0 \% i \mathrm{PrOH}$. Interestingly, the $\mathrm{CD}$ spectra are even more sensitive toward the solvent composition: a change from 41.3 to $41.9 \%$ PrOH already gives rise to $\mathrm{CD}$ of approximately half of the maximal intensity. Moreover, the maximal intensity is reached at $45.0 \% i \mathrm{PrOH}$-further addition of nonsolvent results in a decrease of the CD intensity. In this respect, it should be noted that, on the one hand, UV-vis spectroscopy probes the planarization (and aggregation) process; $\mathrm{CD}$, on the other hand, is sensitive for chiral aggregation. As a consequence, the UV-vis spectra depend on the number of aggregates formed, while the $\mathrm{CD}$ spectra are determined by both the number of aggregates and their chirality and, more in particular, by the angle by which the stacked polymer chains are rotated toward each other. ${ }^{10}$ Therefore, the discrepancy between the evolution of the UV-vis and CD spectra upon decreasing the solvent quality points at a change of the angle between the stacked polymer chains. Initially (i.e., at a low nonsolvent ratio), this angle is rather large, while at higher nonsolvent concentrations a more parallel orientation, which gives rise to bisignate Cotton effects of lower intensity, is obtained. This explains the drop of the CD intensity at higher nonsolvent concentrations: the increase of the fraction of aggregated polymer chains is overcompensated by their smaller individual CD. A similar behavior has already been observed for other chiral (homo)polymers and was attributed to the fact that the addition of a solvent which poorly interacts with the conjugated backbone strengthens the intermolecular $\pi$-interactions, favoring a parallel orientation of the polymer chains.

A decrease of the solvent quality results in a decrease of the emission intensity together with a red-shift (Figure S2). This is fully in line with a planarization and stacking of the polymer chains. The residual emission does not originate from remaining unaggregated polymer chains, as this cannot account for the red-shift. Therefore, the fluorescence must arise from the aggregates.

The possibility to selectively oxidize the BT units in the copolymers was investigated by cyclic voltammetry and chemical oxidation. A pseudoreversible oxidation at $E_{1 / 2} \approx$ $0.9 \mathrm{~V}$ (scan rate $=100 \mathrm{mV} / \mathrm{s}$ ), independent of the length of the OPE, was observed. The fact that oxidation occurs at a much lower potential than that of poly(phenylene ethylene)s and irrespective of the length of the OPE, demonstrates that the BT part is selectively oxidized. The polymer solutions could also be oxidized by a chemical oxidant $\left(\mathrm{FeCl}_{3}\right)$, forming new bands near 700 and $1000 \mathrm{~nm}$ (Figure S3).

From the chiroptical characterization, it can be concluded that, in terms of chiral expression, sensitivity toward external stimuli (e.g., nonsolvent), solubility, and synthetic accessibility, the trimeric OPE gives the best results, and therefore, it was decided to incorporate the carboxylic acid functions in ter(phenylene ethynylene)s. Indeed, since we anticipated that the incorporation of carboxylic acid groups decreases the solubility (which is also the case, see later), we opted to use a functionalized OPE composed of only one phenyl group bearing carboxylic acid functions and at least one alkylfunctionalized phenyl moiety. This excludes the use of a monomeric OPE. A dimeric and tetrameric OPE was not preferred, since the synthesis of such an asymmetric monomer is not straightforward and since it introduces additional problems of regioregularity in the resulting polymer. In addition, the absence of the targeted chiroptical behavior in the related polymer (P4) impeded the use of the tetrameric OPE. For synthetic convenience, the functional group was implemented at the central phenyl group of the trimeric OPE.

Polymers P5a-8a. In a next step, an ester/carboxylic acid function was introduced in the polymers $(\mathbf{P 5} \mathbf{a}-\mathbf{8 a})$. In order to investigate whether the presence of this group affects the (chiral) organization of the copolymers, the influence of the solvent quality on the UV-vis and CD spectra of P5a and P7a was examined (Figure 3).

For these experiments, it appeared not to be necessary to use $i \mathrm{PrOH}$, and hence, methanol was used. For both polymers, chiral aggregation could be induced, but the shape of the spectra differs. While the spectra of $\mathbf{P 7}$ a resemble more or less those of P1-4, the spectra of aggregated P5a lack the sharp, low-energy band with the corresponding Cotton effect. Since this band originates from a (chiral) aggregate of multiple polymer chains and while the broad band with 

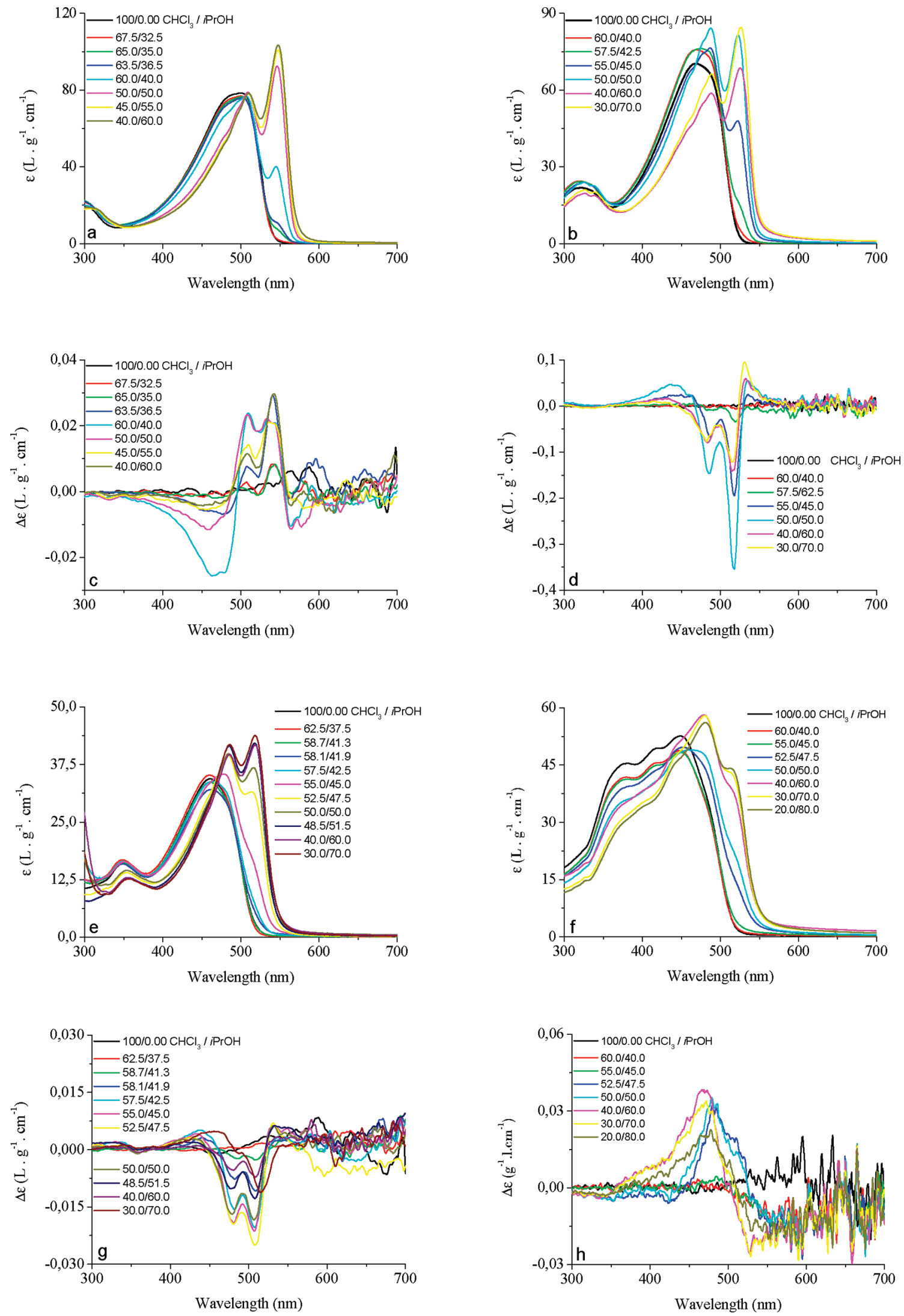

Figure 2. UV-vis spectra of P1 $\left(c=11.2 \times 10^{-3} \mathrm{~g} / \mathrm{L}\right)(\mathrm{a}), \mathbf{P} 2\left(c=12.0 \times 10^{-3} \mathrm{~g} / \mathrm{L}\right)(\mathrm{b}), \mathbf{P} 3\left(c=12.5 \times 10^{-3} \mathrm{~g} / \mathrm{L}\right)(\mathrm{e})$, and $\mathbf{P 4}\left(c=12.5 \times 10^{-3} \mathrm{~g} / \mathrm{L}\right)$ (f) and CD spectra of P1 (c), P2 (d), P3 (g), and P4 (h)

bisignate Cotton effects stems from (an ensemble of) chirally oriented polymer chains, these findings suggest that P5a does not form an aggregated superstructure under poor solvent conditions. This might be correlated with the absence of a melting peak in the DSC experiments. The disappearance of the formation of such a chiral superstructure in P5a can 

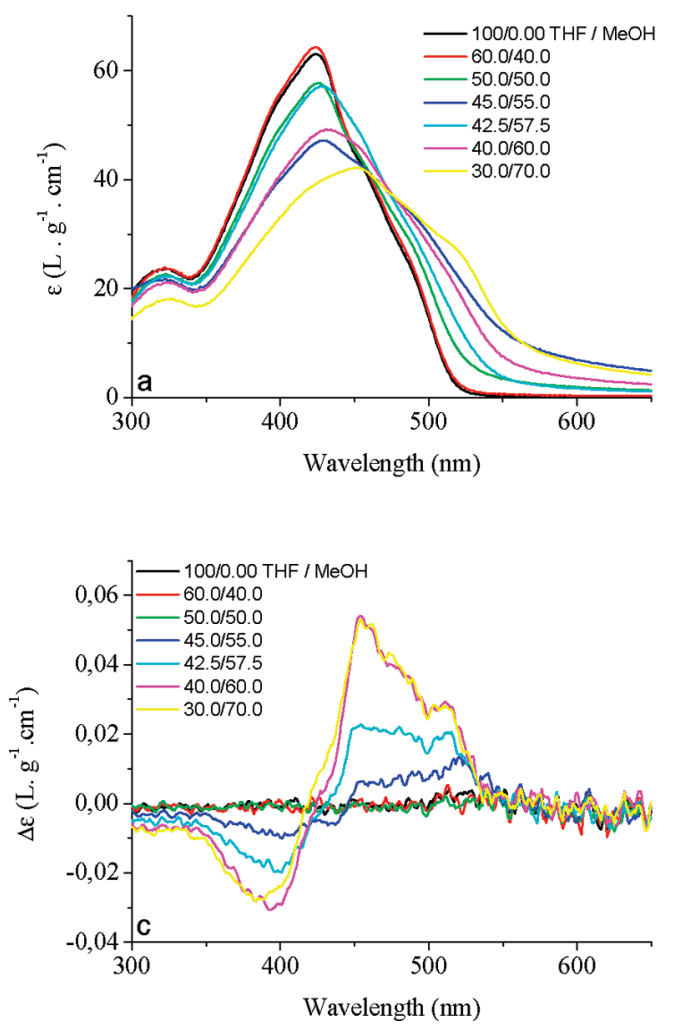
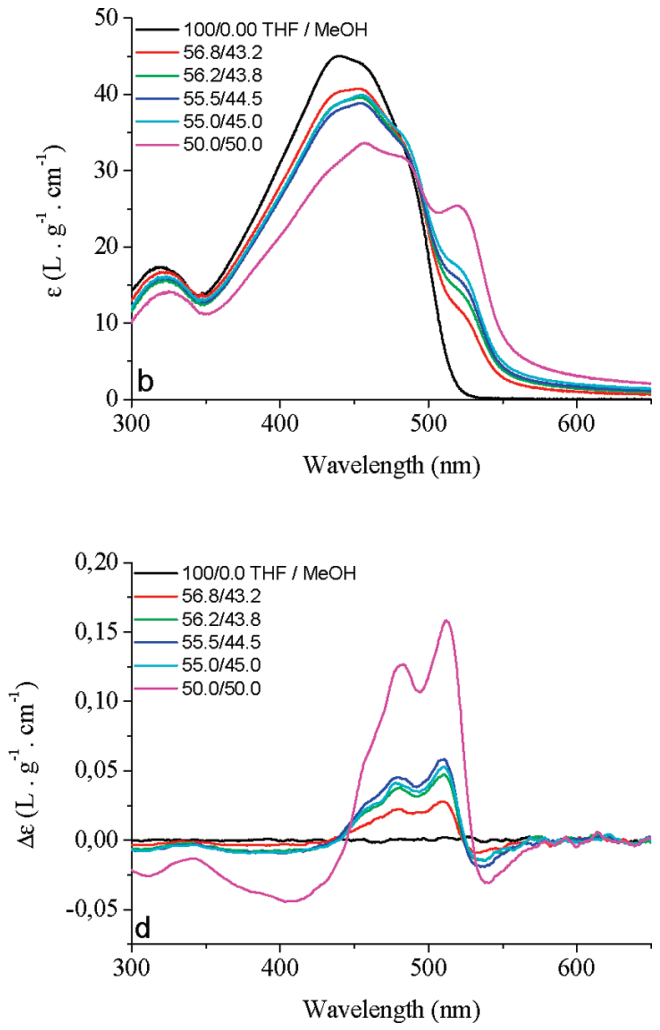

Figure 3. UV-vis spectra of P5a $\left(c=13.5 \times 10^{-3} \mathrm{~g} / \mathrm{L}\right)(\mathrm{a})$ and P7a $\left(c=14.0 \times 10^{-3} \mathrm{~g} / \mathrm{L}\right)(\mathrm{b})$ and CD spectra of P5a (c) and P7a (d).

temptatively be attributed to the presence of the ester functionality close to the polymer backbone, which might disrupt the formation of such a regular structure. Indeed, lamellar aggregation of P5a incorporates the (polar) ester functionality into the alkyl phase. In the case of P7a, an alltrans conformation of the longer alkyl spacer locates the ester function at the periphery of the alkyl phase, which is energetically more favorable.

Polymers $\boldsymbol{P} \mathbf{5} \boldsymbol{b}-\boldsymbol{8 b}$. In a first series of experiments, it was again verified whether the deprotected polymers $\mathbf{P 5 b}-\mathbf{P 8 b}$ show the same chiroptical behavior as their protected counterparts. The solvatochromism experiments (Figure S4) of $\mathbf{P 5 b}-\mathbf{P 8 b}$ again reveal that all polymers show a red-shift but that only $\mathbf{P 7 b}-\mathbf{P 8 b}$ display the low-energy band originating from the superstructure composed of aggregated polymer chains. The quenching of the fluorescence was also more pronounced for the latter polymers. Quite remarkably, no Cotton effects are observed for the chiral $\mathbf{P} 7 \mathbf{b}$ in a poor solvent mixture.

In summary, these experiments show that stacking of the polymer chains can be induced but that in the case of the shorter alkyl spacers the stacking is complicated and no chiral resolvation is present. This might be attributed to the H-bonding of the carboxylic groups, which overwhelms the possible chiral induction of the chiral substituents.

Chiroptical Properties in Solution: Interaction with Additives. Influence of the Additive. Apart from poor solvents, aggregation can also be induced by additives which are able to interact with the carboxylic acid functionality. Especially chiral additives are of interest, as they might induce chiral aggregation of achiral polymers. . $^{211,12}$

A broad range of (a)chiral additives were screened. Therefore, the achiral P8b was dissolved in THF and the additive was added. The samples were allowed to stabilize for $12 \mathrm{~h}$, since preliminary experiments showed that some additives did not instantly interact with the functional groups of the polymers. Among the additives tested (carboxylic acids, alcohols, primary amines, and tertiary amines), only primary amines resulted in aggregation (Figure 4a). In these cases, a clear red-shift can be observed together with an additional shoulder at $514 \mathrm{~nm}$. Clearly, smaller (primary) amines, such as $(S)-(+)$-2-butylamine and $(R)-(-)$-2-amino-1-butanol, induce aggregation at already low base concentrations, while more bulky amines, such as $(L)-(-)-\alpha$-methylbenzylamine, require a higher concentration. Concerning the chiral expression (Figure 4b), the more bulky primary amines resulted in the occurrence of (bisignate) Cotton effects, which implies a transfer of chirality from the amine to the polymer chain upon interaction with the carboxylate functional group. In contrast, the small chiral amines $((S)-(+)$-2-butylamine and $(R)-(-)-2-$ amino-1-butanol), which clearly induce aggregation as shown by UV-vis spectroscopy, are not capable of inducing a twist of the stacking of the polymer chains. Therefore, the bulkiness of the chiral amine seems to be crucial to induce chiral aggregation of the achiral polymer P8b. Emission spectroscopy also confirms the aggregation induced by the (primary) amine, as the fluorescence of the polymer backbone is quenched upon aggregation (Figure $4 \mathrm{c}$ ).

In order to verify whether the aggregation indeed originates from an interaction between the carboxylic acid and amine, $(L)-(-)$ - $\alpha$-methylbenzylamine was added to the esterfunctionalized polymer P8a, which did not change the spectra at all (Figure S5). Therefore, it can be concluded that the aggregation is induced by this interaction. The aggregation induced by the addition of primary amines is likely due to salt formation, followed by H-bonding between the ammonium and (two) carboxylate groups.

Similar experiments were also performed on the chiral polymer P7b. As shown in Figure S6, addition of amines results in aggregation, as eminent from the slight red shift $(10-15 \mathrm{~nm})$ and the occurrence of a very weak, however typical, shoulder at $514 \mathrm{~nm}$ and the quenching of the 

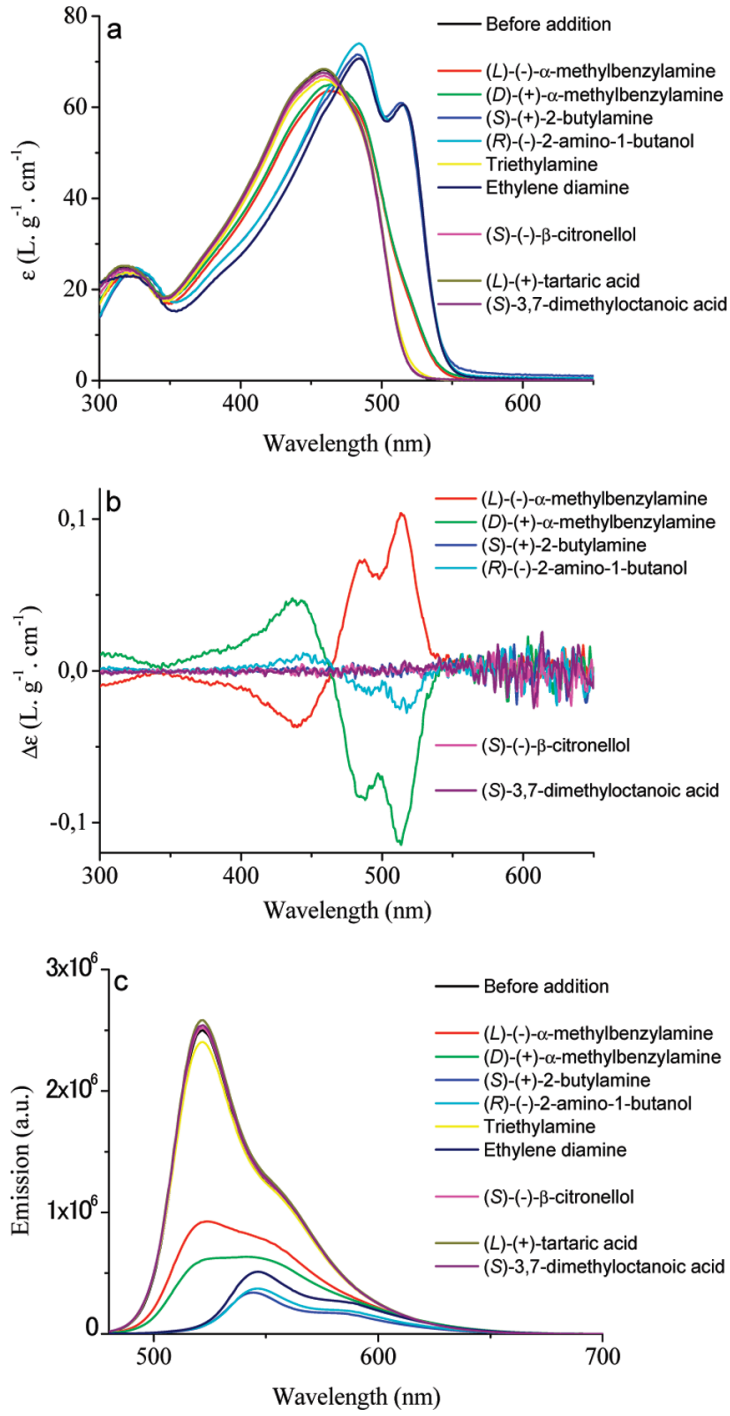

Figure 4. Interaction of different additives $\left(c=2.32 \times 10^{-2} \mathrm{M}\right)$ with P8b in THF solution ( $c=0.0106 \mathrm{~g} / \mathrm{L})$ : (a) UV-vis spectra; (b) CD spectra; (c) emission spectra.

fluorescence (Figure S6). As shown in Figure S4, P7b aggregates upon addition of a poor solvent, but no Cotton effects are observed; that is, no chiral aggregation occurs. On the other hand, addition of benzylamine does induce chiral aggregation, as shown by the (small) CD signal (Figure 5). The possibility to induce chiral aggregation by addition of a primary amine, which breaks the dimerization of the carboxylic acid functionalities, supports the hypothesis that this dimerization is the preventing factor for a chiral stacking of P7b in poor solvent mixtures.

Next, the chiroptical behavior of this chiral polymer upon addition of chiral amines is studied (Figure 5). Therefore, $(L)-(-)-\alpha$-methylbenzylamine and $(D)-(+)-\alpha$-methylbenzylamine were added to $\mathbf{P 7} \mathbf{b}$. To compensate for the experimental error, the experiments were repeated five times and averaged. Clearly, the Cotton effect induced by $(D)-(+)-\alpha$-methylbenzylamine is significantly larger than that with $(L)-(-)$ $\alpha$-methylbenzylamine. It also differs from the sum of the Cotton effect obtained by benzylamine, in which the chiral alkyl groups on the bithiophene invoke the chiral effects, and the inverse of the CD spectra of $(L)-(-)-\alpha$-methylbenzylamine, in which the chirality originates from the chiral amine, demonstrating the diastereomeric interactions.
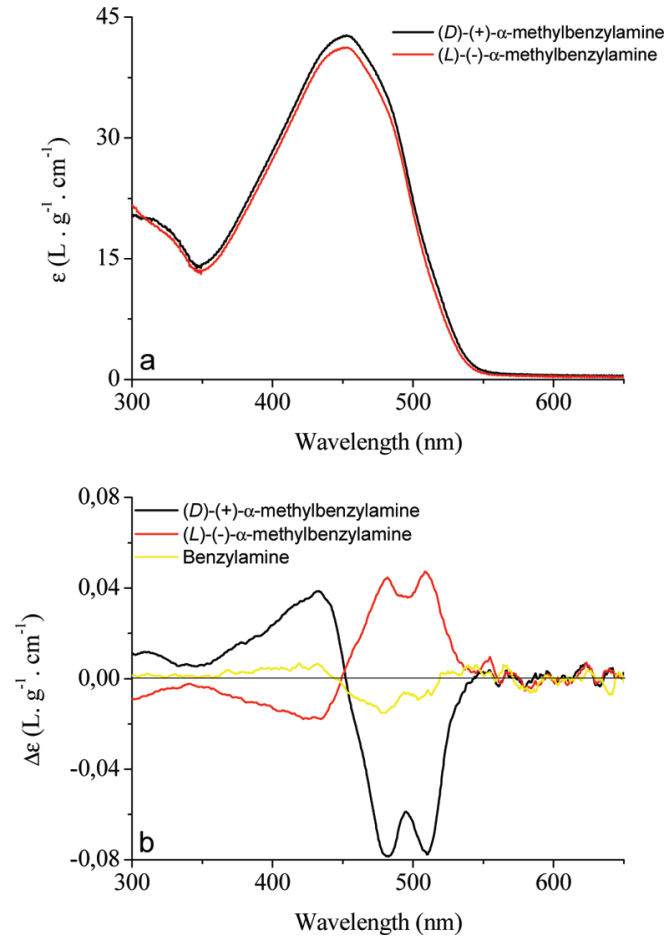

Figure 5. Interaction of different benzylamines $\left(c=9.65 \times 10^{-2} \mathrm{M}\right)$ with P7b in THF ( $c=0.0098 \mathrm{~g} / \mathrm{L}$ ): (a) UV-vis spectra; (b) CD spectra.

Finally, the interaction of amines with $\mathbf{P} \mathbf{6 b}$ was investigated (Figures S7). However, no indication of aggregation was found, as the UV-vis spectra only display a slight redshift, but no typical shoulder near $514 \mathrm{~nm}$. Emission spectroscopy also only shows a slight quenching of the fluorescence but no evidence of a similar aggregate emission band as observed for $\mathbf{P 8 b}$. The decrease in emission intensity can be attributed to clustering of some polymer chains, resulting in traps. The absence of interactions between amines and the functional groups of the polymer in $\mathbf{P} \mathbf{6} \mathbf{b}$ can again be correlated with the polymer structure. Indeed, the carboxylic acid functional groups in $\mathbf{P} \mathbf{6 b}$ are located close to the polymer backbone. As a consequence, in lamellar aggregates, the short alkyl spacer locates the interacting functional groups in the alkyl phase, which is energetically not favorable.

Influence of Amine Concentration. The influence of the amine concentration on the chiroptical properties of $\mathbf{P 8 b}$ was investigated by gradual addition of $(L)-(-)$ - $\alpha$-methylbenzylamine. Prior to the measurements, all samples were allowed to stabilize for $12 \mathrm{~h}$. The added amounts of amine largely exceed the number of carboxylic acid groups $\left(1.6 \times 10^{-5} \mathrm{M}\right)$ present in the polymer solutions. This large discrepancy can be attributed to the very low concentrations and the relative small difference in acidity of a carboxylic acid and an ammonium functionality. As a consequence, the deprotonation of the carboxyl acid by 1 equiv of amine does not result in complete conversion and higher base concentrations are required for a complete deprotonation.

As shown in Figure $6 \mathrm{c}$, addition of $2.50 \times 10^{-6} \mathrm{M}$ of $(L)-(-)-\alpha$-methylbenzylamine leads to a slight quenching of the fluorescence. No red-shift, however, of the fluorescence is observed, nor are any changes in the UV-vis and CD spectra present (Figure 6a and b). This suggests that initial, partial deprotonation induces some clustering of polymer chains, which serve as energy traps, but no aggregation. At $2.50 \times$ $10^{-3} \mathrm{M}$ of base added, also some tailing in the UV-vis spectra can be observed. This is again correlated with the 

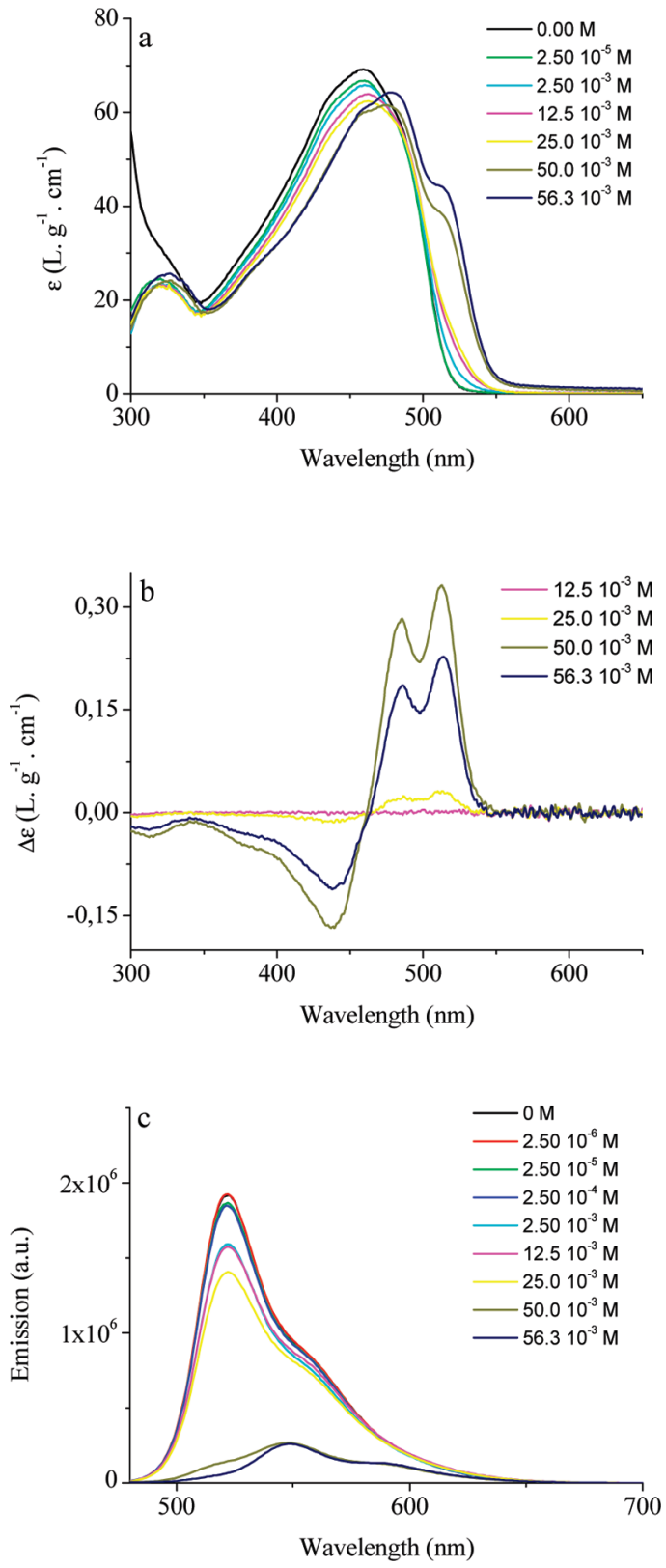

Figure 6. Sensitivity analysis of P8b $(c=0.0106 \mathrm{~g} / \mathrm{L}$ in THF) upon addition of $(L)-(-)$ - $\alpha$-methylbenzylamine: (a) UV-vis spectra; (b) CD spectra; (c) emission spectra.

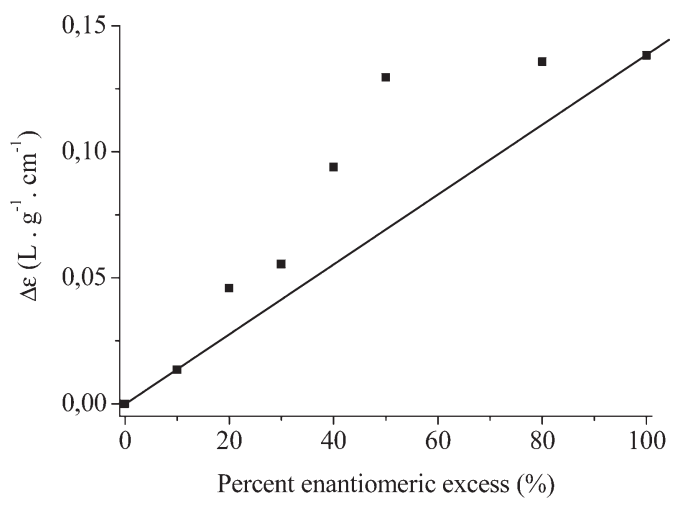

Figure 7. Influence of the enantiomeric excess of $\alpha$-methylbenzylamine $(c=0.1 \mathrm{M})$ on $\Delta \varepsilon$ at $514 \mathrm{~nm}$ of $\mathbf{P 8 b}(c=0.011 \mathrm{~g} / \mathrm{L})$ in THF. The straight line corresponds to a noncooperative behavior and is added as a guide to the eye. a

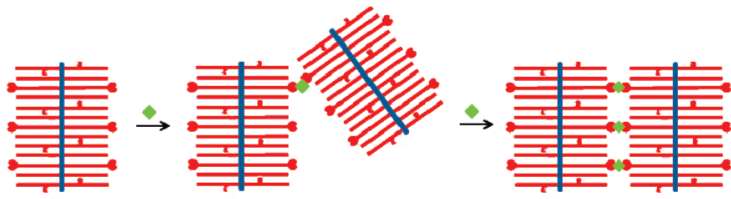

Dissolved

Polymer clusters

Aggregates

b
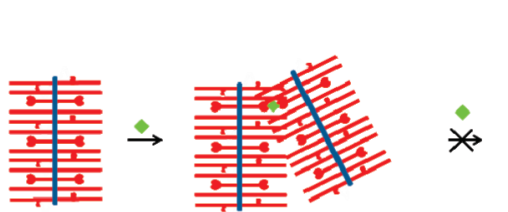

Dissolved

Polvmer clusters

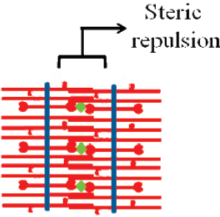

Aggregates

- Carbosțlic acid functional group

$\checkmark=$ Amine base

Figure 8. Schematic presentation of the supramolecular behavior upon interaction with (chiral) amines of (a) $\mathbf{P 7} \mathbf{b}-\mathbf{8 b}$ and (b) $\mathbf{P 5} \mathbf{5}-\mathbf{6} \mathbf{b}$.

clustering, but no aggregation, as the typical aggregation band at $514 \mathrm{~nm}$ and the appearance of a CD signal are still absent. From a concentration of $25.0 \times 10^{-3} \mathrm{M}$ of $(L)-(-)-\alpha-$ methylbenzylamine, the polymer chains start to aggregate, as is eminent from the appearance of a $\mathrm{CD}$ signal. Slight increases of amine concentration $\left(50.0 \times 10^{-3} \mathrm{M}\right)$ result in the appearance of large $\mathrm{CD}$ signals, the aggregation-related absorption band at $514 \mathrm{~nm}$, and red-shifted emission of much smaller intensities - all of which are indications of the formation of aggregates.

Majority Rules. Finally, the influence of the enantiomeric excess (ee) of $(L)-(-)-\alpha$-methylbenzylamine on the intensity of the Cotton effects of $\mathbf{P 8 b}$ was evaluated (majority rules experiment). If $\Delta \varepsilon$ at $514 \mathrm{~nm}$ is evaluated as a function of the ee of the base added (Figure 7), it is clear that a nonlinear relationship is present, demonstrating cooperativity. For instance, at ee $=50$, the $\mathrm{CD}$ signal already equals that in which pure $(L)-(-)-\alpha$-methylbenzylamine was added. A similar behavior has also been observed for poly(thiophene)s upon the addition of chiral nonsolvent. ${ }^{12}$ Moreover, this cooperative behavior also demonstrates that both enantiomers randomly interact with the polymer chains. Indeed, if each polymer chain exclusively interacts with one isomer and if each aggregate is exclusively composed of polymer chains interacting with the same enantiomer, the intensity of the CD signal would linearly scale with the ee, which is clearly not observed.

Supramolecular Behavior. Figure 8 schematically summarizes the supramolecular behavior of $\mathbf{P 5 b}-\mathbf{8 b}$ upon interaction with (chiral) amines. As presented in Figure 8a, $\mathbf{P 7} \mathbf{b}-\mathbf{8 b}$ show the tendency to form polymer clusters at low amine concentrations. This is observed by a slight quenching of the fluorescence and small changes in the shape of the UV-vis spectra. At higher amine concentrations, (chiral) aggregation is induced.

In contrast, $\mathbf{P 5} \mathbf{b} \mathbf{-} \mathbf{6} \mathbf{b}$ only show the formation of polymer clusters even at a high amine concentration, as can be concluded from the UV-vis, CD, and fluorescence spectra. This phenomenon can be understood in terms of the polymer structure. In a lamellar supramolecular structure, the functional groups of $\mathbf{P 5 b}-\mathbf{6 b}$ are partially shielded from their environment, which complicates interactions of multiple chains with the (chiral) amines, allowing only polymer clustering. In contrast, the molecular structure of $\mathbf{P 7 b}-\mathbf{8 b}$ 

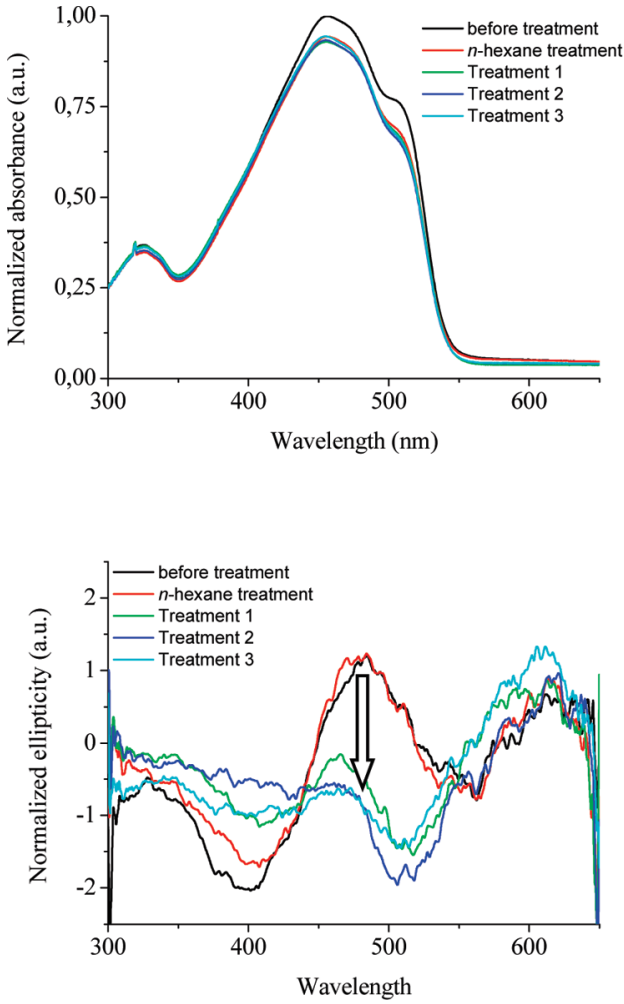
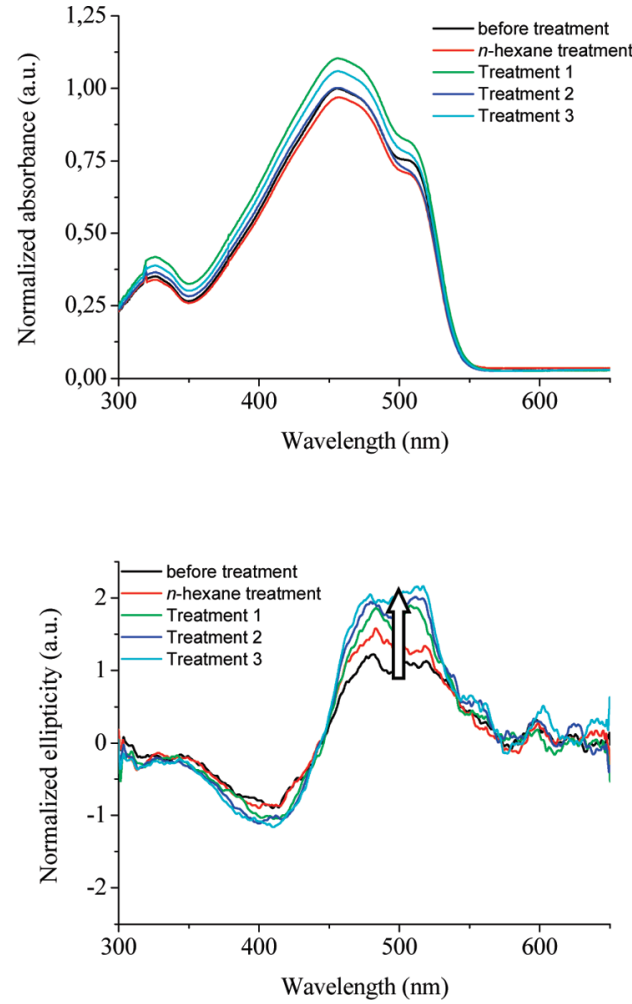

Figure 9. Influence on the circular dichroism upon dipping a film of P7b in a (a) $(L)-(-)$ - $\alpha$-methylbenzylamine solution $(c=0.031 \mathrm{M}$ in $n$-hexane) and a (b) (D)-(-)- $\alpha$-methylbenzylamine solution $(c=0.031 \mathrm{M}$ in $n$-hexane).

facilitates aggregate formation, as the functional groups are located in the periphery of the alkyl phase, allowing a less hindered interaction between the amines and different polymer chains.

Chiroptical Properties in Film. Finally, also the chiroptical properties of films of $\mathbf{P 7} \mathbf{b}$ were evaluated. Therefore, films were spin coated from a THF solution $(3.1 \mathrm{mg} / \mathrm{mL})$. As P7b is chiral, the as-prepared film shows a small bisignate Cotton effect (Figure 9). Next, the films were dipped in solutions of either $(L)-(-)$ - $\alpha$-methylbenzylamine or $(D)-(+)$ - $\alpha$-methylbenzylamine in $n$-hexane, rinsed with $n$-hexane (in order to remove residual amine), and dried. The experiments were repeated three times in order to verify the reproducibility. The interaction with $(L)-(-)$ - $\alpha$-methylbenzylamine results in an inversion of the CD signal (Figure 9a), while exposure to $(D)-(+)-\alpha$-methylbenzylamine increases the magnitude of the CD signal (Figure 9b). Importantly, treatment of the samples with pure $n$-hexane did not affect the CD signal, which excludes solvent-induced annealing effects. These results demonstrate the potential of this polymer as a chiral sensor. Note that the signals in the CD spectra at $\lambda>600 \mathrm{~nm}$, at which wavelength the polymer does not absorb, cannot be due to circular dichroism but probably arise from scattering.

\section{Conclusion}

Different poly(phenylene ethynylene-alt-bithiophene)s with (chiral) substituents were synthesized, and the chiroptical behavior was evaluated by UV-vis and CD spectroscopy. The length of the phenylene ethynylene part was varied (1 to 4 repeating units), revealing a high solvent-sensitive aggregate formation. Next, carboxylic acid-functionalized polymers were prepared. Addition of primary amines results in aggregation; if chiral moieties are present-either in the substituent or from the amine- and depending on the length of the side-chain, circular dichroism can be observed. Moreover, diastereoselectivity and the "majority-rules principle" could be observed. The amineinduced aggregation is a multistep process, in which, first (achiral), clustering occurs and, in a subsequent step, chiral aggregation. Finally, the diastereomeric behavior could be exploited in film by dipping them in a solution of chiral amine.

Acknowledgment. We are grateful to the Katholieke Universiteit Leuven (GOA and Onderzoeksfonds K.U.Leuven/ Research Fund K.U.Leuven), the Fund for Scientific Research (FWO-Vlaanderen), and the Air Force Office of Scientific Research, Air Force Material Command, USAF, for financial support. G.K. is a postdoctoral fellow of the Fund for Scientific Research (FWO-Vlaanderen).

Supporting Information Available: IR spectra of the functionalized polymers, showing the conversion of the ester functional group into a carboxylic acid group. Also, the emission spectra of P1-4, in both their dissolved and aggregated states. The UV-vis spectra of $\mathbf{P 1}-\mathbf{4}$ upon oxidation with $\mathrm{FeCl}_{3}$ are shown. Figures concerning the functionalized polymers are included, showing the solvatochromism of $\mathbf{P 7 b}$ and $\mathbf{P 8 b}$, the interaction of P8a with $(L)-(-)-\alpha$-methylbenzylamine, and the interaction of $\mathbf{P} \mathbf{6 b}$ and $\mathbf{P 7} \mathbf{b}$ with different (chiral) amines. Finally, ${ }^{1} \mathrm{H}$ NMR and ${ }^{13} \mathrm{C}$ NMR spectra of the new compounds are presented. This material is available free of charge via the Internet at http://pubs.acs.org.

\section{References and Notes}

(1) (a) Halkyard, C. E.; Rampey, M. E.; Kloppenburg, L.; StuderMartinez, S. L.; Bunz, U. H. F. Macromolecules 1998, 31, 86568659. (b) Fiesel, R.; Halkyard, C. E.; Rampey, M. E.; Kloppenburg, L.; Studer-Martinez, S. L.; Scherf, U.; Bunz, U. H. F. Macromol. Rapid Commun. 1999, 20, 107-111.

(2) (a) Bidan, G.; Guillerez, S.; Sorokin, V. Adv. Mater. 1996, 8, 157160. (b) De Cremer, L.; Verbiest, T.; Koeckelberghs, G. Macromolecules 2008, 41, 568-578. (c) Van den Bergh, K.; Cosemans, I.; Verbiest, T.; Koeckelberghs, G. Macromolecules 2010, 43, 3794-3800. 
(3) (a) Ishikawa, M.; Maeda, K.; Yashima, E. J. Am. Chem. Soc. 2002, 124, 7448-7458. (b) Hase, Y.; Ishikawa, M.; Muraki, R.; Maeda, K.; Yashima, E. Macromolecules 2006, 39, 6003-6008. (c) Saito, M. A.; Maeda, K.; Onouchi, H.; Yashima, E. Macromolecules 2000, 33, 46164618. (d) Vangheluwe, M.; Verbiest, T.; Koeckelberghs, G. J. Polym. Sci., Part A: Polym. Chem. 2008, 46, 4817-4829. (e) Kim, J.; McQuade, D. T.; McHugh, S. K.; Swager, T. M. Angew. Chem., Int. Ed. 2000, 39, 3868-3872. (f) Kim, I. B.; Dunkhorst, A.; Gilbert, J.; Bunz, U. H. F. Macromolecules 2005, 38, 4560-4562. (g) Ramey, M. B.; Hiller, J. a.; Rubner, M. F.; Tan, C.; Schanze, K. S.; Reynolds, J. R. Macromolecules 2005, 38, 234-243. (h) Wang, F.; Bazan, G. C. J. Am. Chem. Soc. 2006, 128, 15786-15792. (i) Liu, X.; Zhou, X.; Shu, X.; Zhu, J. Macromolecules 2009, 42, 7634-7637. (j) Jiang, H.; Taranekar, P.; Reynolds, J. R.; Schanze, K. S. Angew. Chem., Int. Ed. 2009, 48, 4300-4316.

(4) (a) Roux, C.; Bergeron, J.-Y.; Leclerc, M. Makromol. Chem. 1993, 194, 869-877. (b) Roux, C.; Leclerc, M. Chem. Mater. 1994, 6, 620624. (c) Leclerc, M.; Faïd, K. Adv. Mater. 1997, 9, 1087-1094.

(5) (a) Langeveld-Voss, B. M. W.; Beljonne, D.; Shuai, Z.; Janssen, R. A. J.; Meskers, S. C. J.; Meijer, E. W.; Brédas, J.-L. Adv. Mater. 1998, 10, 1343-1348. (b) Oda, M.; Nothofer, H.-G.; Scherf, U.; Šunjić, V.; Richter, D.; Regenstein, W.; Neher, D. Macromolecules 2002, 35, 6792-6798. (c) Koeckelberghs, G.; Vangheluwe, M.; Samyn, C.;
Persoons, A.; Verbiest, T. Macromolecules 2005, 38, 5554-5559. (d) Vandeleene, S.; Van den Bergh, K.; Verbiest, T.; Koeckelberghs, G. Macromolecules 2008, 41, 5123-5131. (e) Babudri, F.; Colangiuli, D.; Di Bari, L.; Farinola, G. M.; Omar, O. H.; Naso, F.; Pescitelli, G. Macromolecules 2006, 39, 5206-5212.

(6) Vangheluwe, M.; Verbiest, T.; Koeckelberghs, G. Macromolecules 2008, 41, 1041-1044.

(7) France, V.; Mangel, T.; Müllen, K. Macromolecules 1998, 31, $2447-2453$

(8) (a) Mangel, T.; Eberhardt, A.; Scherf, U.; Bunz, U. H. F.; Müllen, K. Macromol. Rapid Commun. 1995, 16, 571-580. (b) Egbe, D. A. M.; Sell, S.; Ulbricht, C.; Birckner, E.; Grummt, U.-W. Macromol. Chem. Phys. 2004, 205, 2105-2115.

(9) Kukula, H.; Veit, S.; Godt, A. Eur. J. Org. Chem. 1999, 1, 277-286.

(10) (a) Circular Dichroic Spectroscopy, Exciton Coupling in Organic Stereochemistry; Harada, N., Nakanishi, K., Eds.; Oxford University Press: Oxford, 1983. (b) Circular Dichroism and Linear Dichroism; Rodger, A., Nordén, B., Eds.; Oxford University Press: Oxford, 1997.

(11) (a) Yoneyama, H.; Tsujimoto, A.; Goto, H. Macromolecules 2007, 40, 5279-5283. (b) Goto, H. Macromolecules 2007, 40, 1377-1385.

(12) Langeveld-Voss, B. M. W.; Waterval, R. J. M.; Janssen, R. A. J.; Meijer, E. W. Macromolecules 1999, 32, 227-230. 